A. INOUE

KODAI MATH. $I$.

9 (1986), 134-160

\title{
SOME EXAMPLES EXHIBITING THE PROCEDURES OF RENORMALIZATION AND GAUGE FIXING. SCHWINGER-DYSON EQUATIONS OF FIRST ORDER
}

\author{
By Atsushi InOUE
}

\begin{abstract}
In this note, a 'definition' of the useful but notorious Feynman measure corresponding to bilinear Lagrangeans with 'singular' coefficients is given through functional derivative equations. Especially, the procedures of renormalization and gauge fixing are clarified at the equation level.
\end{abstract}

\section{Contents}

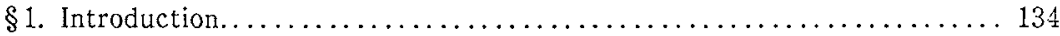

§2. Derivation of the renormalized S-D equation corresponding to (I) ... 137

$\S 3$. Construction of an explicit solution of renormalized S-D equations (I) 139

$\$ 4$. Derivation of the renormalized and gauge fixed S-D equation

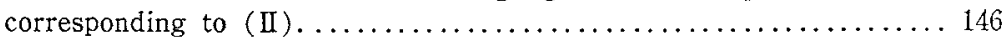

$\$ 5$. Construction of an explicit solution of renormalized S-D equations (II) .149 Appendix A. Derivations of formal S-D equations. ................ 152

Appendix B. The Feynman propagator. ..................... 156

Appendix $C$. Generalizations. .............................. 157

\section{$\S 1$. Introduction.}

When a mathematician wants to study the non-relativistic quantum theory, he may find the Schrödinger equation which is considered as governing the dynamics of non-relativistic quantum mechanics. Without taking physics into account, he may study properties of the solutions of the Schrödinger equation. Moreover, if he is lucky, he may say something to physicists.

In spite of this, when he wants to study the quantum field theory (Q.F.T.), he may be a little bit confused because there exists no mathematically well defined equation.

From our point of view, the so-called Schwinger-Dyson (S-D) equation, derived by using the functional derivative, seems a candidate of the equation governing the dynamics of the Q.F.T., but in general, it contains infinities be-

Received May 23, 1985 
fore 'renormalization'. (cf, for example, C. Itzykson \& J.B. Zuber [13], I.M. Gelfand [9].)

In this paper, we take up two 'formal' S-D equations of first order. The points in our discussions are: (1) we may define directly from such formally given S-D equations, the equations called 'renormalized' ones, which do not contain 'apparent' infinities and (2) we may construct explicit solutions of them without solving corresponding classical field equations. Moreover, the meaning of the gauge fixing is considered at the equation level in the second example.

In other words, using the functional derivative equation (F.D.E.), we may 'define' the quantity represented formally by notorious Feynman measure.

For physicists, the first order S-D equations are regarded as trivial objects to consider, so no one presents the renormalized S-D equation explicitly. In general, physicists calculate something what they want by the analogy of the procedure which they have already used with success ((?) even the success of calculations in QED is now doubted by physicists themselves) and there is no need of the exact representation of the 'equation' itself because it does not help their calculations.

But for mathematicians, the equation itself has a meaning in general if it is well-defined and without explicit representation of it, we have no thought of 'solving' it.

Now, we describe two 'formal' S-D equations whose derivations will be explained in Appendix A.

(I) Find a functional $Z=Z(p, u)$ of real functions $p(t)$ and $u(x, t), t \in \boldsymbol{R}$, $x \in \boldsymbol{R}^{3}$ such that it satisfies the following 'equations'.

$$
\begin{gathered}
\left(\frac{d^{2}}{d t^{2}}+\omega_{0}^{2}\right) \frac{\delta Z}{\delta p(t)}=\frac{i}{h} p(t) Z-\lambda \int_{R^{3}} \delta(x) \frac{\delta Z}{\delta u(x, t)} d x, \\
\square \frac{\delta Z}{\delta u(x, t)}=\frac{\imath}{h} u(x, t) Z-\lambda \delta(x) \frac{\delta Z}{\delta p(t)}
\end{gathered}
$$

and

$$
Z(0,0)=1
$$

Here $\delta(x)$ is Dirac's delta function, $\square=\frac{\partial^{2}}{\partial t^{2}}-\Delta, \Delta=\sum_{j=1}^{3} \frac{\partial^{2}}{\partial x_{j}^{2}}, \frac{\delta}{\delta p(t)}$ and $\frac{\delta}{\delta u(x, t)}$ stand for the functional (or Frechet-Volterra) derivatives with respect to $p(t)$ and $u(x, t)$ whose definitions will be given in $\S 2$.

(II) Find a functional $Z=Z(\boldsymbol{p}, \boldsymbol{B})$ of real vector functions $\boldsymbol{p}(t)=\left(p_{1}(t), p_{2}(t)\right.$, $\left.p_{3}(t)\right), t \in \boldsymbol{R}$ and $\boldsymbol{B}(x, t)=\left(B^{0}(x, t), B^{ \pm}(x, t), B^{2}(x, t), B^{3}(x, t)\right), \quad(x, t) \in \boldsymbol{R}^{3} \times \boldsymbol{R}=\boldsymbol{R}^{4}$ such that it satisfies the following 'equations' :

$$
\begin{aligned}
\left(\frac{d^{2}}{d t^{2}}+\omega_{0}^{2}\right) \frac{\delta Z}{\delta p_{j}(t)}= & \frac{i}{h} p_{j}(t) Z \\
& +e \int_{R^{3}} \delta(x)\left(\frac{\partial}{\partial t} \frac{\delta Z}{\delta B^{3}(x, t)}-\frac{\partial}{\partial x^{3}} \frac{\delta Z}{\delta B^{0}(x, t)}\right) d x
\end{aligned}
$$




$$
\begin{aligned}
\Delta \frac{\delta Z}{\delta B^{0}(x, t)}= & \frac{i}{h} B^{0}(x, t) Z+\frac{\partial}{\partial t} \frac{\partial}{\partial x^{k}} \frac{\delta Z}{\delta B^{k}(x, t)}+e \frac{\partial}{\partial x^{k}} \delta(x) \frac{\delta Z}{\delta p_{k}(t)}, \quad \text { (II .2) } \\
\square \frac{\delta Z}{\delta B^{j}(x, t)}= & \frac{i}{h} B^{j}(x, t) Z \\
& -\frac{\partial}{\partial x^{j}}\left(\frac{\partial}{\partial x^{k}} \frac{\delta Z}{\delta B^{k}(x, t)}-\frac{\partial}{\partial t}-\frac{\delta Z}{\delta B^{0}(x, t)}\right)-e \delta(x) \frac{d}{d t} \frac{\delta Z}{\delta p_{j}(t)}
\end{aligned}
$$

and

$$
Z(0,0)=1
$$

Hereafter, we use Einstein's convention, that is, $\frac{\partial}{\partial x^{k}} \frac{\delta Z}{\delta B_{k}(x, t)}$ stands for $\sum_{k=1}^{3}$ $\frac{\partial}{\partial x^{k},} \frac{\delta Z}{\delta B^{k}(x, t)}$ etc.

Our results are: (1) Instead of the equation ( 1.1 ) which contains 'apparent' infinity in the last term, we may derive in $\$ 2$ the renormalized one given as

$$
\left(\frac{d^{2}}{d t^{2}}+\omega_{0}^{2}-i \frac{\lambda^{2}}{4 \pi}\left|-\frac{d}{d t}\right|\right) \frac{\delta Z}{\delta p(t)}=\frac{i}{h} p(t) Z-\frac{i}{h} \lambda \int_{R^{3}} \delta(x)\left(\square_{F}^{-1} u\right)(x, t) d x Z \text {. ( I . 1) }
$$

Where, the operator $\left|\frac{d}{d t}\right|$ will be defined by (2.9).

The term $\frac{\lambda^{2}}{4 \pi}\left|\frac{d}{d t}\right|$ appears as the counter term of the term contains infinity, i.e. $\lambda^{2} \int_{R^{3}}|\xi|^{-2} d \xi$, which is dicarded under the name of renormalization. $\square_{F}^{-1}$ is the inverse operator of $\square$ with the kernel $G_{F}^{B}(x, t)$ given by

$$
G_{F}^{B}(x, t)=(2 \pi)^{-4} \int_{R^{4}} \frac{e^{2 x \xi-2 \tau \tau}}{-\tau^{2}+|\xi|^{2}-i 0} d \xi d \tau
$$

whose meaning will be explained in Appendix B. Then we may give an explicit solution of $(I .1)_{R},(I .2)$ and ( I .3) in $\S 3$.

(2) As the last term in equation (II.1) contains 'apparent' infinity, we renormalize it as

$$
\left(\frac{d^{2}}{d t^{2}}+\omega_{0}^{2}-i \frac{e^{2}}{6 \pi}\left|\frac{d}{d t}\right|^{3}\right) \frac{\delta Z}{\delta p_{j}(t)}=\frac{i}{h} p_{j}(t) Z-e \frac{\imath}{h} \int_{R^{3}} \delta(x)\left(\square F^{-1} \frac{\partial}{\partial t} B^{j}\right)(x, t) d x Z
$$

The term $-\frac{e^{2}}{6 \pi}\left|\frac{d}{d t}\right|^{3}$ appears as the counter term of $\frac{2}{3} e^{2} \int_{R^{3}}|\xi|^{-1} d \xi$. This term 
and also the term $\frac{1}{3} e^{2} \int_{R^{3}} d \xi$ are deleted in deriving (II.1) $)_{R, g}$ by eliminating the 'variable' $B^{0}(x, t)$. After reformulating the problem (II) as in $\S 4$, we may construct an explicit solution of them in $\$ 5$.

In Appendix $\mathrm{C}$, we discuss the possibility of extending these results to more realistic cases. For example, the so-called Zachariasen model is solved there. There appears Heaviside function $\theta(x)$ instead of $\delta(x)$ as a 'singular' coefficient in a bilinear Lagrangean. And other examples are discussed there.

\section{§2. Derivation of the renormalized S-D equation corresponding to $(I)$.}

First of all, we define functional dervatives. Let $E$ be a function space on $\boldsymbol{R}^{n}$ containing $C_{0}^{\infty}\left(\boldsymbol{R}^{n}\right)$ (=the space of infinitely differentiable functions with compact support) whose dual space, denoted by $E^{\prime}$, being contained in $D^{\prime}\left(\boldsymbol{R}^{n}\right)$ (=the space of distributions). $\langle$,$\rangle stands for the duality between E$ and $E^{\prime}$ and it represents also other dualities which will be explained for each instances.

Definition 2.1. Let $Z=Z(f)$ be a functional on $E$. If at $f \in E$, there exists $D Z(f) \in E^{\prime}$ such that for all $g \in E$

$$
\left.\frac{d}{d \varepsilon} Z(f+\varepsilon g)\right|_{\varepsilon=0}=\langle D Z(f), g\rangle,
$$

then $Z$ is called differentiable at $f$ and $D Z(f)$ is called the functional (or FrechetVolterra) derivative of $Z$ at $f$. We represent formally the right hand side of (2.1) as

$$
\langle D Z(f), g\rangle=\int_{R^{n}} \frac{\delta Z(f)}{\delta f(x)} g(x) d x .
$$

$\frac{\delta Z(f)}{\delta f(x)}$ is sometimes called the functional derivative of $Z$ by $f$ at $x$ or of $Z$ at $f(x)$.

If a functional $Z$ depends on many 'variables' $\left(f_{1}, f_{2}, \cdots, f_{m}\right)$, then we may define 'partial' functional derivative $\frac{\delta Z\left(f_{1}, f_{2}, \cdots, f_{m}\right)}{\delta f_{j}(x)}$ analogously. (cf. V. Volterra [19].)

Even if the independent variables of 'variables' are different, for example, $Z(p, u), p=p(t)$ and $u=u(x, t)$, we may define $\frac{\delta Z}{\delta p(t)}$ and $\frac{\delta Z}{\delta u(x, t)}$ in the same manner as above.

DEFINITION 2.2. Let $Z$ be a differentiable functional on $E$. If for each $h_{1} \in E$, $\left\langle D Z(f), h_{1}\right\rangle$ is differentiable as a functional of $f, Z$ is called two times differer tiable at $f$ and its two times derivative $D^{2} Z(f)$ is given by 


$$
\left.\frac{d}{d \varepsilon}\left\langle D Z\left(f+\varepsilon h_{2}\right), h_{1}\right\rangle\right|_{\varepsilon=0}=\left\langle D^{2} Z(f), h_{1} \otimes h_{2}\right\rangle .
$$

Here, $\langle$,$\rangle appeared in the right-hand side of (2.3) stands for the duality between$ $E=E\left(\boldsymbol{R}^{2 n}\right)$ and $E^{\prime}=E^{\prime}\left(\boldsymbol{R}^{2 n}\right)$ and $\left(h_{1} \otimes h_{2}\right)(x, y)=h_{1}(x) h_{2}(y), x, y \in \boldsymbol{R}^{n}$. Moreover, by the kernel theorem of $\mathrm{L}$. Schwartz [18], we may represent the right-hand side of (2.3) as

$$
\begin{aligned}
\left\langle D^{2} Z(f), h_{1} \otimes h_{2}\right\rangle & =\int_{R^{2 n}} \frac{\delta^{2} Z(f)}{\delta f(x) \delta f(y)} h_{1}(x) h_{2}(y) d x d y \\
& =\int_{R^{2 n}} \frac{\delta^{2} Z(f)}{\delta f(y) \delta f(x)} h_{1}(x) h_{2}(y) d x d y .
\end{aligned}
$$

Higher order derivatives as well as higher order partial derivatives of a functional with many 'variables' are defined analogously.

Remark 2.3. The use of the equation containing the functional derivatives appeared at least in the famous paper of E. Hopf [11]. But there exists scarce paper treating the existence of the solution of F.D.E., except for statistical studies of Navier-Stokes equations, e.g. M.D. Donskar \& J.L. Lions [6], P. Levy [14], E. Hopf, C. Foias [7, 8], etc.

By the definition of functional derivatives, the last term in ( I.1) contains the product of distributions, which is undefined in general. This is the meaning of 'apparent' infinity. Using ( I.2), we want to redefine the last term in ( 1.1 ) by specifying and discarding the infinity.

Applying $\square_{F}^{-1}$ to (I.2) and inserting it to (I.1), we get

$$
\begin{gathered}
\left(\frac{d^{2}}{d t^{2}}+\omega_{0}^{2}\right) \frac{\delta Z}{\delta p(t)}-\lambda^{2}\left\langle\delta(\cdot),\left(\square_{F}^{-1}\left(\delta(\cdot) \frac{\delta Z}{\delta p(\cdot)}\right)\right)(\cdot, t)\right\rangle \\
=\frac{i}{h} p(t) Z-\frac{\imath}{h} \lambda\left\langle\delta(\cdot),\left(\square_{F}^{-1} u\right)(\cdot, t)\right\rangle Z .
\end{gathered}
$$

Here $\langle v, w\rangle$ stands for the formal expression $\int v(x) w(x) d x$. and $\delta(x)$ is treated as if it is a function.

Now, we consider the following operator

$$
\left(A_{\lambda}^{\infty} v\right)(t)=\left(\frac{d^{2}}{d t^{2}}+\omega_{0}^{2}\right) v(t)-\lambda^{2}\left\langle\delta(\cdot),\left(\square_{F}^{-1}(\delta v)\right)(\cdot, t)\right\rangle .
$$

Renormalization in this case is to define the finite operator $A_{\lambda}^{R}$ by subtracting the infinity from the last term in (2.6).

Let $\rho_{\varepsilon}(x)$ be a function defined by $\rho_{\varepsilon}(x)=\varepsilon^{-3} \rho(x / \varepsilon), \quad \rho(x)=\rho(|x|) \in C_{0}^{\infty}\left(\boldsymbol{R}^{3}\right)$, $\rho(x) \geqq 0$ and $\int_{\boldsymbol{R}^{3}} \rho(x) d x=1$. Then $\rho_{\varepsilon}(x) \rightarrow \delta(x)$ as $\varepsilon \rightarrow 0$ in $D^{\prime}\left(\boldsymbol{R}^{3}\right)$. We define an operator $A_{\lambda}^{\Sigma}$ as 


$$
\left(A_{\hat{\lambda}}^{\varepsilon} v\right)(t)=\left(\frac{d^{2}}{d t^{2}}+\omega_{0}^{2}\right) v(t)-\lambda^{2}\left\langle\rho_{\varepsilon}(\cdot),\left(\square_{F}^{-1}\left(\rho_{\varepsilon} v\right)\right)(\cdot, t)\right\rangle .
$$

Applying Fourier transform and using Plancherel's formula, we get

$$
\begin{aligned}
\left(A_{\lambda}^{\varepsilon} \hat{v}\right)(\tau)= & \frac{1}{\sqrt{2 \pi}} \int_{R} e^{i t \tau}\left(A_{\lambda}^{\varepsilon} v\right)(t) d t \\
= & \left(-\tau^{2}+\omega_{0}^{2}\right) \hat{v}(\tau)+\frac{\lambda^{2}}{4 \pi^{2}} \int_{R^{3}} \frac{\left|\hat{\rho}_{\varepsilon}(\xi)\right|^{2}}{\tau^{2}-|\xi|^{2}+i 0} d \xi \hat{v}(\tau) \\
= & \left(-\tau^{2}+\omega_{0}^{2}\right) \hat{v}(\tau) \\
& +\frac{\lambda^{2}}{4 \pi^{2}}\left[\int_{R^{3}}\left|\hat{\rho}_{\varepsilon}(\xi)\right|^{2}\left(\frac{1}{\tau^{2}-|\xi|^{2}+i 0}+\frac{1}{|\xi|^{2}}\right) d \xi-\int_{R^{3}} \frac{\left|\hat{\rho}_{\varepsilon}(\xi)\right|^{2}}{|\xi|^{2}} d \xi\right] \hat{v}(\tau)
\end{aligned}
$$

Making $\varepsilon \rightarrow 0$ and neglecting the last term which diverges to $\infty$, we may show readily that the right hand side of (2.7) converges to

$$
\left\{\left(-\tau^{2}+\omega_{0}^{2}\right)-i \frac{\lambda^{2}}{4 \pi}|\tau|\right\} \hat{v}(\tau)
$$

Defining an operator $A_{\lambda}^{R}$ as

$$
\left(A_{\lambda}^{R} v\right)(t)=\left(\frac{d^{2}}{d t^{2}}+\omega_{0}^{2}-\imath \frac{\lambda^{2}}{4 \pi}\left|\frac{d}{d t}\right|\right) v(t)
$$

we have

$$
A_{\lambda}^{R} \frac{\delta Z}{\delta p(t)}=\frac{\imath}{h} p(t) Z-\frac{\imath}{h} \lambda\left\langle\delta,\left(\square \bar{F}^{1} u(\cdot, t)\right\rangle Z\right.
$$

Here the operator $\left|\frac{d}{d t}\right|$ stands for

$$
\left|\frac{d}{d t}\right| v(t)=\frac{1}{\sqrt{2 \pi}} \int_{R}|\tau| e^{-i t \tau} \hat{v}(\tau) d \tau \quad \text { for } \quad v \in S(\boldsymbol{R}) .
$$

Remark. There is no special reason why the infinity of the above form is subtracted. But we think, at least for the time being, that it is intuitively 'natural' to define the renormalized operator as above.

\section{§. Construction of an explicit solution of renormalized S-D equations (I).}

Though $Z$ is a functional of 'variables' $p(t)$ and $u(x, t)$, we may not consider those as equal weight ones because the independent variables of $p$ and those of $u$ are different. So we want to separate the $x$-variable of $u(x, t)$. To do so, first of all, we consider the case where the 'variable' $u(x, t)$ has its support with respect to $x$ in a bounded domain $\Omega$ in $\boldsymbol{R}^{3}$ containing the orgin and having the smooth boundary $\partial \Omega$. 
For such $\Omega$, we consider the following problem: Find a functional $Z_{\Omega}=$ $Z_{\Omega}(p(\cdot), u(\cdot, \cdot))$, for supp $u(\cdot, t) \subset \Omega$ satisfying

$$
\begin{gathered}
A_{\lambda}^{R} \frac{\delta Z_{\Omega}}{\delta p(t)}=\frac{i}{h} p(t) Z_{\Omega}-\frac{i}{h} \lambda\left\langle\delta(\cdot),\left(\square{ }_{F, \Omega}^{-1} u\right)(\cdot, t)\right\rangle Z_{\Omega}, \\
\square-\frac{\delta Z_{\Omega}}{\delta u(x, t)}=\frac{i}{h} u(x, t) Z_{\Omega}-\lambda \delta(x) \frac{\delta Z_{\Omega}}{\delta p(t)}
\end{gathered}
$$

and

$$
Z_{\Omega}(0,0)=1
$$

Here $\square_{F, \Omega}^{-1}$ is the operator with kernel

$$
G_{F, \Omega}^{B}(x, y, t)=\sum_{l=1}^{\infty} \frac{w_{l}(x) w_{l}(y)}{2 \mu_{l}}\left\langle\theta(t) e^{-\eta_{\mu} t}+\theta(-t) e^{\imath^{\mu} \mu^{t}}\right) .
$$

Here the following notations are used: $\left\{\mu_{l}\right\},\left\{w_{l}(x)\right\}$ are eigenvalues and eigenfunctions of

$$
-\Delta w_{l}(x)=\mu_{l}^{2} w_{l}(x) \text { in } \Omega \text { with }\left.w_{l}\right|_{\partial \Omega}=0
$$

Using the expansion $u(x, t)=\sum_{l=1}^{\infty} u_{l}(t) w_{l}(x)$ for $u$ with supp $u(\cdot, t) \subset \Omega$, we may identify $u(x, t)$ as $\left\{u_{l}(t)\right\}$.

Then we may rewrite the problem as follows: Find a functional $Z_{\Omega}=$ $Z_{\Omega}\left(p(\cdot), u_{1}(\cdot), u_{2}(\cdot), \cdots\right)$ such that

$$
\begin{aligned}
& A_{\lambda}^{R} \frac{\delta Z_{\Omega}}{\delta p(t)}=\frac{i}{h} p(t) Z_{\Omega}-\frac{l}{h} \lambda \sum_{l=1}^{\infty} \rho_{l}\left(B_{l, F}^{-1} u_{l}\right)(t) Z_{\Omega}, \\
& B_{l} \frac{\delta Z_{\Omega}}{\delta u_{l}(t)}=\frac{i}{h} u_{l}(t) Z_{\Omega^{-}}-\lambda \rho_{l} \frac{\delta Z_{\Omega}}{\delta p(t)} \quad \text { for } \quad l=1,2, \cdots
\end{aligned}
$$

and

$$
Z_{\Omega}(0,0)=1
$$

Here $A_{\lambda}^{R}$ is given in $\S 2, B_{l}=\frac{d^{2}}{d t^{2}}+\mu_{l}^{2}, B_{l, F}^{-1}$ is defined as

$$
B_{l, F}^{-1}=\frac{1}{2 \mu_{l}}\left(\theta(t) e^{-\imath \mu_{l} t}+\theta(-t) e^{\imath \mu^{\ell}}\right)
$$

and $\rho_{l}=w_{l}(0)=\left\langle\delta, w_{l}\right\rangle$.

To solve (3.5)-(3.7), we regard these equations as if they form a system of partial differential equations of first order with infinite independent variables. That is, we construct a solution $u(x, y)=u\left(x, y_{1}, y_{2}, \cdots\right)$ of the following equations. (Here $p(t)$ and $u_{l}(t)$ corresponds to $x$ and $y_{l}$, respectively. Be careful, we abbuse symbols a little bit because of the lack of them.)

$$
a_{\lambda} u_{x}=\frac{i}{h} x u-\frac{i}{h} \lambda \sum_{l=1}^{\infty} \rho_{l} b_{l}^{-1} y_{l} u
$$




$$
\begin{gathered}
b_{l} u_{y_{l}}=\frac{i}{h} y_{l} u-\lambda \rho_{l} u_{x} \quad \text { for } \quad l=1,2, \cdots \\
u(0,0)=1
\end{gathered}
$$

where we regard 'scalars' $a_{\lambda}$ and $b_{l}$ as if they correspond to operators $A_{\lambda}^{R}$ and $B_{l}$, respectively.

By simple calculations, we have a following solution for the above problem.

$$
\begin{aligned}
u(x, y)= & \exp \left[\frac{i}{2 h} a_{\lambda}^{-1} x^{2}+\frac{i}{2 h} \sum_{l=1}^{\infty}\left(\frac{y_{l}}{b_{l}}\right)^{2}+\frac{i}{2 h} \lambda^{2} a_{\lambda}^{-1}\left(\sum_{l=1}^{\infty} \frac{\rho_{l} y_{l}}{b_{l}}\right)^{2}\right. \\
& \left.-\frac{i}{h} \lambda a_{\lambda}^{-1} x\left(\sum_{l=1}^{\infty} \frac{\rho_{l} y_{l}}{b_{l}}\right)\right] .
\end{aligned}
$$

Here we assume implicity that (i) $a_{k}$ is invertible, and (ii) $y=\left(y_{1}, y_{2}, \cdots\right) \in \boldsymbol{R}^{\infty}$ must satisfy $\sum_{l=1}^{\infty}\left(\frac{y_{l}}{b_{l}}\right)^{2}<\infty$ and $\sum_{l=1}^{\infty} \frac{\rho_{l} y_{l}}{b_{l}}<\infty$. Assuming moreover, $\lim _{i \rightarrow 0} a_{k}=a_{0}$, where $a_{0}$ corresponds to $A_{0}=\frac{d^{2}}{d t^{2}}+\omega_{0}^{2}$, we get

$$
\begin{aligned}
& \lim _{\lambda \rightarrow 0} u_{x, x}(0)=\frac{i}{h} a_{0}^{-1}, \\
& \lim _{\lambda \rightarrow 0} u_{y_{l} y_{k}}(0)=\frac{i}{h} \delta_{l k} b_{l}^{-1}
\end{aligned}
$$

and

$$
\lim _{\lambda \rightarrow 0} u_{x y_{l}}(0)=0 .
$$

Remembering that $\left\{w_{l}\right\}$ forms a orthonormal basis in $L^{2}(\Omega)$, we have the following formulas at least formally :

$$
\left\langle\square \square_{, \Omega 2}^{-1} v, v\right\rangle_{\Omega}=\sum_{l=1}^{\infty}\left\langle B_{l, F^{-1}}^{-1} v_{l}, v_{l}\right\rangle
$$

where $v(\cdot, t)=\sum_{l=1}^{\infty} v_{l}(t) w_{l}(\cdot)$, and $\operatorname{supp} v(\cdot, t) \subset \Omega$.

$$
\begin{aligned}
& \left\langle\delta, \square \bar{F}^{-1}, Q\right\rangle_{\varrho}=\left(\square-\square_{F}^{-1}, \varrho v\right)(0, t)=\sum_{l=1}^{\infty} \rho_{l}\left(B_{l, F}^{-1} v_{l}\right)(t) \\
& \left\langle\left(A_{\lambda}^{R}\right\rangle^{-1}\left\langle\delta, \square_{F}^{-1}, \Omega v\right\rangle_{Q},\left\langle\delta, \square_{F}^{-1}, \Omega v_{\Omega}\right\rangle_{\Omega}\right\rangle=\left\langle\left(A_{\lambda}^{R}\right)^{-1} \sum_{l=1}^{\infty} \rho_{l} B_{l, F}^{-1} v_{l}, \sum_{l=1}^{\infty} \rho_{l} B_{l, F}^{-1} v_{l}\right\rangle
\end{aligned}
$$

where $\langle w(\cdot, t), v(\cdot, t)\rangle_{\Omega}=\int_{\Omega} w(x, t) v(x, t) d x$ and $\langle p(\cdot), q(\cdot)\rangle=\int p(t) q(t) d t$.

For the sake of completeness, we derive the above formulas. Consider the equation

$$
\square u(x, t)=v(x, t) \quad \text { in } \Omega \times(-\infty, \infty)
$$


with $\left.u(x, t)\right|_{\partial \Omega}=0$ and $\left.v(x, t)\right|_{\partial \Omega}=0$. Using the expansion of $v(x, t)=$ $\sum_{l=1}^{\infty} v_{l}(t) w_{l}(x)$ and $u(x, t)=\sum_{l=1}^{\infty} u_{l}(t) w_{l}(x)$, we have

$$
B_{l} u_{l}(t)=v_{l}(t) \quad \text { for any } l \text {. }
$$

So, applying $B_{l, F}^{-1}$ to both sides and summing up with respect to $l$, we have the formula (3.16). (3.17) comes from the fact $\left\langle\delta, w_{l}\right\rangle=w_{l}(0)=\rho_{l}$ where we consider $w_{l}(x) \in C_{0}\left(\boldsymbol{R}^{3}\right)$ by extending 0 outside of $\Omega$. (3.18) follows from (3.16) and (3.17) if $\left(A_{\lambda}^{R}\right)^{-1}$ is applicable to $\left\langle\delta, \square \bar{F}_{,}^{-1} \Omega\right\rangle_{\Omega}$.

From (3.12) and above formulas, we may put

$$
\begin{aligned}
Z_{\Omega}(p, u)= & \exp \left[\frac{i}{2 h}\left\langle\left(A_{\lambda}^{R}\right)^{-1} p, p\right\rangle+\frac{i}{2 h}\left\langle\square_{F}^{-1}, \Omega, u\right\rangle_{\Omega}\right. \\
& +\frac{i}{2 h} \lambda^{2}\left\langle\left(A_{\lambda}^{R}\right)^{-1}\left\langle\delta, \square_{F, \Omega}^{-1} u\right\rangle_{\Omega},\left\langle\delta, \square_{F}^{-1}, \Omega\right\rangle_{\Omega}\right\rangle \\
& \left.-\frac{i}{h} \lambda\left\langle\left\langle\delta, \square_{F}^{-1}, \Omega\right\rangle_{\Omega},\left(A_{\lambda}^{R}\right)^{-1} p\right\rangle\right] .
\end{aligned}
$$

Then, we may check easily that (3.19) gives a solution of (3.1)-(3.3) by the same calculation which will be given below.

Apart from the convergence of $\square \square_{F, \Omega}^{-1}$ to $\square_{F}^{-1}$ when $\Omega$ tends to $\boldsymbol{R}^{3}$, we may define a functional $Z(p, u)$ as

$$
\begin{aligned}
Z(p, u)= & \exp \left[\frac{i}{2 h}\left\langle\left(A_{\lambda}^{R}\right)^{-1} p, p\right\rangle+\frac{i}{2 h}\left\langle\square_{F}^{-1} u, u\right\rangle\right. \\
& +\frac{i}{2 h} \lambda^{2}\left\langle\left(A_{\lambda}^{R}\right)^{-1}\left\langle\delta, \square_{F}^{-1} u\right\rangle,\left\langle\delta, \square_{F}^{-1} u\right\rangle\right\rangle \\
& \left.-\frac{i}{h} \lambda\left\langle\left(A_{\lambda}^{R}\right)^{-1} p,\left\langle\delta, \square_{F}^{-1} u\right\rangle\right\rangle\right] .
\end{aligned}
$$

Now, we want to give an exact meaning to the expression above.

LEMMA 3.1. (a) The operator $A_{\lambda}^{R}(\lambda>0)$ is invertible and $\left(A_{\lambda}^{R}\right)^{-1}$ sends $H^{-1}(\boldsymbol{R})$ boundedly to $H^{1}(\boldsymbol{R})$. So the bracket $\left\langle\left(A_{\lambda}^{R}\right)^{-1} p, p\right\rangle$ is well-defined for $p \in H^{1}(\boldsymbol{R})$.

(b) For $u \in S\left(\boldsymbol{R}^{4}\right), \square_{F}^{-1} u$ belongs to $S^{\prime}\left(\boldsymbol{R}^{4}\right)$. Moreover, if $u \in C_{0}^{\infty}\left(\boldsymbol{R}^{4}\right)$, then $\lim _{\varepsilon \rightarrow 0}\left\langle\rho_{\varepsilon}(\cdot),\left(\square_{F}^{-1} u\right)(\cdot, t)\right\rangle$ exists in $H^{-1}(\boldsymbol{R})$ and denoted by $\left\langle\delta(\cdot),\left(\square_{F}^{-1} u\right)(\cdot, t)\right\rangle$ or $\left\langle\delta, \square_{F}^{-1} u\right\rangle(t)$.

Here, $H^{k}(\boldsymbol{R})$ stands for the Sobolev space of order $k, S\left(\boldsymbol{R}^{4}\right)$ denotes the space of rapidly decreasing functions and $S^{\prime}(\boldsymbol{R})$ means the space of tempered distributions.

Proof. By the definition of the operator $A_{\lambda}^{R}$, the claim (a) is rather trivial because for $\lambda>0,-\tau^{2}-\frac{i \lambda^{2}}{4 \pi}|\tau|+\omega_{0}^{2} \neq 0$ for any $\tau \in R$. Other statements are proved 
in Appendix B.

The above lemma guarantees that the functional $Z(p, u)$ is well-defined on $H^{-1}(\boldsymbol{R}) \times C_{0}^{\infty}\left(\boldsymbol{R}^{4}\right)$.

Calculating the functional derivatives of $Z$, we have

$$
\frac{\delta Z(p, u)}{\delta p(t)}=\left[\frac{i}{h}\left(A_{\lambda}^{R}\right)^{-1} p(t)-\frac{i}{h} \lambda\left(A_{\lambda}^{R}\right)^{-1}\left\langle\delta, \square_{F}^{-1} u(\cdot, t)\right\rangle\right] Z(p, u)
$$

and

$$
\begin{aligned}
\frac{\delta Z(p, u)}{\delta u(x, t)}= & {\left[\frac{i}{h} \square_{F}^{-1} u(x, t)+\frac{i}{h} \lambda^{2} \square_{F}^{-1}\left(\delta(x)\left(A_{\lambda}^{R}\right)^{-1}\left\langle\delta, \square_{F}^{-1} u(\cdot, t)\right\rangle\right)\right) } \\
& \left.-\frac{i}{h} \lambda \square_{F}^{-1}\left(\delta\left(A_{\lambda}^{R}\right)^{-1} p\right)(x, t)\right] Z(p, u) .
\end{aligned}
$$

From these equations, we have easily the following theorem.

THEOREM I. The functional $Z(p, u)$ given by $(3.20)$ is well-defined on $H^{-1}(\boldsymbol{R})$ $\times C_{0}^{\infty}\left(\boldsymbol{R}^{4}\right)$, is infintely differentiable and satisfies (I.1) $)_{R},(\mathrm{I} .2)$ and (I.3).

Remark. Concerning the uniqueness of the solution, see the remark at the end of this section.

From above, we may calculate 2-points functions readily.

Corollary I.1.

$$
\begin{aligned}
& G_{F, \lambda}^{R, H}(t-s)=i h \frac{\delta^{2} Z(0,0)}{\delta p(t) \delta p(s)} \\
& =(2 \pi)^{-1} \int_{R} \frac{e^{-\imath(t-s) \tau}}{-\tau^{2}+\omega_{0}^{2}-i \frac{\lambda^{2}}{4 \pi}|\tau|} d \tau=\left(A_{\lambda}^{R}\right)^{-1}(t-s) . \\
& G_{F, B}^{R, B}((x, t),(y, s))=i h \frac{\delta^{2} Z(0,0)}{\delta u(x, t) \delta u(y, s)} \\
& =G_{F}^{B}(x-y, t-s) \\
& +\lambda^{2} \int_{R^{8}} G_{F^{\prime}, \lambda}^{R, H}\left(t_{2}-t_{1}\right) \delta\left(x_{1}\right) \delta\left(x_{2}\right) G_{F}^{B}\left(x_{1}-x, t_{1}-t\right) G_{F}^{B}\left(x_{2}-y, t_{2}-s\right) d x_{1} d x_{2} d t_{1} d t_{2} \\
& =(2 \pi)^{-4} \int_{R^{4}} \frac{e^{-\imath(t-s) \tau+\imath(x-y) \xi}}{\tau^{2}-|\xi|^{2}+i 0} d \tau d \xi \\
& +\lambda^{2}(2 \pi)^{-5} \int_{R^{7}} \frac{e^{-\imath(t-\beta) \tau-\imath x \xi-\imath y \eta}}{\left(-\tau^{2}+\omega_{0}^{2}-i \frac{\lambda^{2}}{4 \pi}|\tau|\right)\left(\tau^{2}-|\xi|^{2}+i 0\right)\left(\tau^{2}-|\eta|^{2}+i 0\right)} d \tau d \xi d \eta
\end{aligned}
$$




$$
\begin{aligned}
G_{F, \lambda}^{R, M}(t,(y, s)) & =i h \frac{\delta^{2} Z(0,0)}{\delta p(t) \delta u(y, s)} \\
& \left.=-\lambda \int_{R^{4}} G_{F, H}^{R}, t_{1}-t\right) \delta\left(x_{1}\right) G_{F^{\prime}}^{B}\left(x_{1}-y, t_{1}-s\right) d x_{1} d t_{1} \\
& =-\lambda(2 \pi)^{-3} \int_{R^{4}} \frac{e^{i(t-s) \tau+\imath y \xi}}{\left(-\tau^{2}+\omega_{0}^{2}-\imath \frac{\lambda^{2}}{4 \pi}|\tau|\right)\left(\tau^{2}-|\xi|^{2}+i 0\right)} d \tau d \xi .
\end{aligned}
$$

From these equations, we have

COROLLARY I.2.

$$
\begin{gathered}
\lim _{\lambda \rightarrow 0} \frac{\delta^{2} Z(0,0)}{\delta p(t) \delta p(s)}=\frac{\imath}{h} G_{F}^{H}(t-s), \\
\lim _{\lambda \rightarrow 0} \frac{\delta^{2} Z(0,0)}{\delta u(x, t) \delta u(y, s)}=\frac{\imath}{h} G_{F}^{B}(x-y, t-s), \\
\lim _{\lambda \rightarrow 0} \frac{\delta^{2} Z(0,0)}{\delta u(x, t) \delta p(s)}=0 .
\end{gathered}
$$

In order to calculate all Green functions which will be given below, we prepare

Lemma 3.2. Put $f(x, y)=\exp \left[-i\left((a / 2) x^{2}+c x y+(b / 2) y^{2}\right)\right]$. Then

$$
\begin{gathered}
\left.\quad\left(\frac{\partial}{\partial x}\right)^{n}\left(\frac{\partial}{\partial y}\right)^{m} f(x, y)\right|_{x=y=0}=0 \quad \text { for } n+m=\text { odd } \\
\left.i^{n+m}\left(\frac{\partial}{\partial x}\right)^{2 n}\left(\frac{\partial}{\partial y}\right)^{2 m} f(x, y)\right|_{x=y=0} \\
=\sum_{k=0}^{m i n(n, m)}\{(2 n) !(2 m) ! /(n-k) !(m-k) !(2 k) !\}(a / 2)^{n-k} c^{2 k}(b / 2)^{m-k}, \\
\left.i^{n+m+1}\left(\frac{\partial}{\partial x}\right)^{2 n+1}\left(\frac{\partial}{\partial y}\right)^{2 m+1} f(x, y)\right|_{x=y=0} \\
=\sum_{k=0}^{\min (n, m)}\{(2 n+1) !(2 m+1) ! /(n-k) !(m-k) !(2 k+1) !\}(a / 2)^{n-k} c^{2 k+1}(b / 2)^{m-k}
\end{gathered}
$$

Proof. These follows from Bell's formula for the derivatives of composite functions.

The following formula is also well-known.

LEMMA 3.3. Put $S(\eta)=\frac{1}{2} \int K(x, y) \eta(x) \eta(y) d x d y$ with a sutable symmetric 
kernel.

$$
\begin{gathered}
\left.\frac{\delta^{n} \exp \left(-\frac{i}{h} S(\eta)\right)}{\delta \eta\left(x_{1}\right) \cdots \delta \eta\left(x_{n}\right)}\right|_{\eta=0}=0 \quad \text { for } n=\text { odd } \\
\left.(i h)^{n} \frac{\delta^{2 n} \exp \left(-\frac{i}{h} S(\eta)\right)}{\delta n\left(x_{1}\right) \cdots \delta \eta\left(x_{2 n}\right)}\right|_{\eta=0}=\sum_{c o m b}^{2 n} K\left(x_{\imath_{1}}, y_{\jmath_{1}}\right) \cdots K\left(x_{\imath_{n}}, y_{\jmath_{n}}\right)
\end{gathered}
$$

where $\sum_{\text {comb }}^{2 n}$ indicates the summ over all $(2 n) ! /\left(2^{n} n\right)$ ways of wrating $1,2, \cdots, 2 n$ as $n$ distinct unordered pairs $\left(i_{1}, j_{1}\right) \cdots\left(i_{n}, j_{n}\right)$, and we denote it for brevity by $\sum_{c o m b}^{2 n}(K(\cdot, \cdot))^{n}$.

Applying these, we have

COROLLARY I.3. Put

$$
\begin{aligned}
& G^{(n, m)}\left(t_{1}, \cdots t_{n},\left(y_{1}, s_{1}\right) \cdots\left(y^{m}, s_{m}\right)\right) \\
& \quad=(i h)^{n+m} \frac{\delta^{n+m} Z(0,0)}{\delta p\left(t_{1}\right) \cdots \delta p\left(t_{n}\right) \delta u\left(y_{1}, s_{1}\right) \cdots \delta u\left(y_{m}, s_{m}\right)} .
\end{aligned}
$$

Then we get

$$
\begin{aligned}
& G^{(n, m)}\left(t_{1}, \cdots, t_{n},\left(y_{1}, s_{1}\right) \cdots\left(y_{m}, s_{m}\right)\right)=0 \quad \text { for } n+m=\text { odd } . \\
& G^{(2 n, 2 m)}\left(t_{1}, \cdots, t_{2 n},\left(y_{1}, s_{1}\right) \cdots\left(y_{2 m}, s_{2 m}\right)\right) \\
& =\sum_{k=0}^{\min (n, m)}\{(2 n) !(2 m) ! /(n-k) !(m-k) !(2 k) !\} 2^{n+m-2 k} \\
& \left.\left\{\sum_{\text {comb }}^{2(n-k)}\left(G_{F, \lambda}^{R, H}(\cdot, \cdot)\right)^{n-k}\right\}\left(\sum_{c o m b}^{2 k}\left(G_{F, \lambda}^{R, M}(\cdot, \cdot)\right)^{2 k}\right\}\left\{\sum_{c o m b}^{2(m-k)} G_{F, \lambda}^{R, B}(\cdot, \cdot)\right)^{m-k}\right\} . \\
& G^{(2 n+1,2 m+1)}\left(t_{1}, \cdots, t_{2 n+1},\left(y_{1}, s_{1}\right) \cdots\left(y_{2 m+1}, s_{2 m+1}\right)\right) \\
& =\sum_{k=0}^{\min (n, m)}\{(2 n+1) !(2 m+1) ! /(n-k) !(m-k) !(2 k+1) !\} 2^{n+m-2 k} \\
& \left\{\sum_{\mathrm{comb}}^{2(n-k)}\left(G_{F}^{R}, H(\cdot, \cdot)\right)^{n-k}\right\}\left(\sum_{\mathrm{comb}}^{2 k+1}\left(G_{F}^{R}, \lambda_{\lambda}(\cdot, \cdot)\right)^{2 k+1}\right\}\left\{\left\{\sum_{\operatorname{comb}}^{2(m-k)} G_{F}^{R, B}, \lambda(\cdot, \cdot)\right)^{m-k}\right\} \cdot
\end{aligned}
$$

Using L-S-Z formula (see, the formula (16.81) in p 149 of J.D. Bjorken-S.D. Drell [4]), we may define $T_{\lambda}^{B}(|\xi|)$ as

$$
\begin{aligned}
& 2 \pi i \delta(|\xi|-|\eta|) T_{\lambda}^{B}(|\eta|) \\
& =(2 \pi)^{-1} \int_{R^{8}} e^{-\imath x_{\xi}+i t \tau} e^{-\imath y r_{\imath}+2 s \sigma} \square(x, t) \square(y, s) G_{F}^{R, B},\left.\lambda((x, t),(y, s)) d x d t d y d s\right|_{\xi=;}
\end{aligned}
$$


where $\square_{(x, t)}=\frac{\partial^{2}}{\partial t^{2}}-\Delta_{x}$ and $\square(y, s)=\frac{\partial^{2}}{\partial s^{2}}-\Delta_{y}$.

The right hand side is calculated easily and gives

$$
T_{\lambda}^{B}(k)=-\frac{\lambda^{2}}{2 k\left\{-k^{2}+\omega_{0}^{2}-i \frac{\lambda^{2}}{4 \pi} k\right\}} \quad \text { for } k>0 .
$$

This is essentially equal to the transition matrix for bosons calculated in A. Arai [3] using the Hamiltonian formalism.

Remark. We are not sure, for the time being, whether (I.1)-(I.3) has at most two solutions. (Because there exists at least two choice in defining the Feynman propagator, that is, as in Appendix $\mathrm{B}$, we may define another $E(x, t)$ replacing $-i \varepsilon$ by $+i \varepsilon$. But this choice is fixed by the behavior of the two point function when $\lambda \rightarrow 0$ by Corollary I.2.)

But if the formula (3.20) affords an unique solution of (I.1)-(I.3) after fixing the behaviour of two points functions for $\lambda \rightarrow 0$, then, comparing the expression (A.2) in Appendix A, we have 'defined' the 'renormalized complex canonical ensemble' represented by Feynman measure.

\section{§4. Derivation of the renormalized and gauge fixed S-D equations corresponding to $(\mathrm{II})$.}

Differentiating both sides of (II.2) by $t$, we have

$$
\frac{\partial}{\partial t} \Delta \frac{\delta Z}{\delta B^{0}(x, t)}=\frac{\imath}{h} \frac{\partial}{\partial t} B^{0}(x, t) Z+\frac{\partial^{2}}{\partial t^{2}} \frac{\partial}{\partial x^{j}} \frac{\delta Z}{\delta B^{2}(x, t)}+e \frac{\partial}{\partial x^{3}} \delta \frac{d}{d t} \frac{\delta Z}{\delta p_{j}(t)} \text {. }
$$

Applying $\frac{\partial}{\partial x^{3}}$ to both sides of (II.3) and summing up with respect to $j$, we get

$$
\frac{\hat{o}^{2}}{\partial t^{2}} \frac{\partial}{\partial x^{3}} \frac{\delta Z}{\delta B^{\jmath}(x, t)}-\frac{\partial}{\partial t} \Delta \frac{\delta Z}{\delta B^{0}(x, t)}=\frac{i}{h} \frac{\partial}{\partial x^{\jmath}} B^{\jmath}(x, t) Z-e \frac{\partial}{\partial x^{j}} \delta \frac{d}{d t} \frac{\delta Z}{\delta p_{j}(t)} .
$$

Adding (4.1) and (4.2), we have

$$
\left(\frac{\partial}{\partial t} B^{0}(x, t)+\frac{\partial}{\partial x^{k}} B^{k}(x, t)\right) Z=0 .
$$

Inserting the relations (4.1) and (4.3) into (II.3), we obtain

$$
\begin{aligned}
& \square \frac{\delta Z}{\delta B^{\jmath}(x, t)}+\frac{\partial}{\partial x^{j}} \frac{\partial}{\partial x^{k}} \frac{\delta Z}{\delta B^{k}(x, t)} \\
& +\frac{\partial}{\partial x^{j}}(-\Delta)^{-1}\left\{-\frac{i}{h} \frac{\partial}{\partial x^{k}} B^{k}(x, t) Z+\frac{\partial}{\partial t^{2}} \frac{\partial}{\partial x^{k}} \frac{\delta Z}{\delta B^{k}(x, t)}+e \frac{\partial}{\partial x^{k}} \delta \frac{d}{d t} \frac{\delta Z}{\delta p_{k}(t)}\right\}
\end{aligned}
$$




$$
=\frac{i}{h} B^{\jmath}(x, t) Z-e \delta(x) \frac{\delta Z}{\delta p_{j}(t)} .
$$

Using the relation $(-\Delta)(-\Delta)^{-1}=I d$, we may rewrite (4.4) as

$$
\begin{gathered}
\square\left(\delta_{j k}+\frac{\partial}{\partial x^{j}}(-\Delta)^{-1} \frac{\partial}{\partial x^{k}}\right) \frac{\delta Z}{\delta B^{k}(x, t)}-\frac{i}{h} \frac{\partial}{\partial x^{j}}(-\Delta)^{-1} \frac{\partial}{\partial x^{k}} B^{k}(x, t) Z \\
=\frac{i}{h} B^{\jmath}(x, t) Z-e\left(\delta_{j k}+\frac{\partial}{\partial x^{j}}(-\Delta)^{-1} \frac{\partial}{\partial x^{k}}\right) \delta(x) \frac{d}{d t} \frac{\delta Z}{\delta p_{k}(t)} .
\end{gathered}
$$

On the other hand, differentiating both sides of (II.1) by $t$ and using (4.2) and (4.3), we have

$$
\begin{aligned}
\left(\frac{d^{2}}{d t^{2}}+\omega_{0}^{2}\right) \frac{d}{d t} \frac{\delta Z}{\delta p_{j}(t)} & \\
= & \frac{i}{h} \frac{d}{d t} p_{j}(t) Z+e\left\langle\delta, \frac{\partial^{2}}{\partial t^{2}}\left(\delta_{j k}+\frac{\partial}{\partial x^{j}}(-\Delta)^{-1} \frac{\partial}{\partial x^{k}}\right) \frac{\delta Z}{\delta B^{k}(\cdot, t)}\right\rangle \\
& -e \frac{i}{h}\left\langle\delta, \frac{\partial}{\partial x^{j}}(-\Delta)^{-1} \frac{\partial}{\partial x^{k}} B^{k}(x, t)\right\rangle Z+e^{2}\left\langle\delta, \frac{\partial}{\partial x^{j}}(-\Delta)^{-1} \frac{\partial}{\partial x^{k}} \delta \frac{d}{d t} \frac{\delta Z}{\delta p_{k}(t)}\right\rangle
\end{aligned}
$$

Using the relation $\square_{F}^{-1} \square=I d$, we insert the relation (4.5) into the second term of the right-hand side of (4.6). Then we get

$$
\begin{gathered}
\left(\frac{d^{2}}{d t^{2}}+\omega_{0}^{2}\right) \frac{d}{d t} \frac{\delta Z}{\delta p_{j}(t)}+e^{2}\left\langle\delta,\left\{\delta_{j k}+\Delta \square_{F}^{-1}\left(\delta_{j k}+\frac{\partial}{\partial x^{j}}(-\Delta)^{-1} \frac{\partial}{\partial x^{k}}\right)\right\} \delta \frac{d}{d t} \frac{\delta Z}{\delta p_{k}(t)}\right\rangle \\
=\frac{i}{h} \frac{d}{d t} p^{j}(t) Z-e \frac{i}{h}\left\langle\delta,\left\{\delta_{j k}+\Delta \square_{F}^{-1}\left(\delta_{j k}+\frac{\partial}{\partial x^{j}}(-\Delta)^{-1} \frac{\partial}{\partial x^{k}}\right)\right\} B^{k}(\cdot, t)\right\rangle Z
\end{gathered}
$$

Now, we consider the following system of ordinary differential operators.

$$
\left(A_{e}^{\infty} v\right)^{\jmath}(t)=\left(\frac{d^{2}}{d t^{2}}+\omega_{0}^{2}\right) v^{\jmath}(t)+e^{2}\left\langle\delta,\left\{\delta_{j k}+\Delta \square_{F}^{-1}\left(\delta_{j k}+\frac{\partial}{\partial x^{j}}(-\Delta)^{-1} \frac{\partial}{\partial x^{k}}\right)\right\} \delta(\cdot) v^{k}(t)\right\rangle .
$$

for $\boldsymbol{v}(t)=\left(v^{1}(t), v^{2}(t), v^{3}(t)\right)$.

Let $\rho_{\varepsilon}(x)$ be defined as in $\S 2$. We define an operator $A_{e}^{\Sigma}$ as

$$
\left(A_{e}^{\varepsilon} \boldsymbol{v}\right)^{\jmath}(t)=\left(\frac{d^{2}}{d t^{2}}+\omega_{0}^{2}\right) v^{\jmath}(t)+e^{2}\left\langle\rho_{\varepsilon},\left\{\boldsymbol{\delta}_{j k}+\Delta \square_{F}^{-1}\left(\boldsymbol{\delta}_{j k}+\frac{\partial}{\partial x^{j}}(-\Delta)^{-1} \frac{\partial}{\partial x^{k}}\right)\right\} \rho_{s}(\cdot) v^{k}(t)\right\rangle
$$

Calculating as before, we get

$$
\left(A_{e}^{\varepsilon} \hat{\boldsymbol{v}}\right)^{\jmath}(\tau)=-\left(1+\frac{2}{3} \frac{e^{2}}{4 \pi^{2}} \int_{R^{3}} \frac{\left|\hat{\rho}_{\varepsilon}(\xi)\right|^{2}}{|\xi|^{2}} d \xi\right) \tau^{2} \hat{v}^{\jmath}(\tau)
$$




$$
\begin{aligned}
& +\frac{2}{3} \frac{e^{2}}{4 \pi^{2}} \int_{R^{3}}\left(\frac{1}{\tau^{2}-|\xi|^{2}+i 0}+\frac{1}{|\xi|^{2}}\right)\left|\hat{\rho}_{\varepsilon}(\xi)\right|^{2} d \xi \cdot \tau^{2} \hat{v}^{j}(\tau) \\
& +\left(\omega_{0}^{2}+\frac{1}{3} \frac{e^{2}}{4 \pi^{2}} \int_{R^{3}}\left|\hat{\rho}_{\varepsilon}(\xi)\right|^{2} d \xi\right) \hat{v}^{\gamma}(\tau) .
\end{aligned}
$$

From here on, the term $1 / 3$ appears several times because we assume implicitly that in our problem all directions in $\boldsymbol{R}^{3}$ have same weights.

Discarding the terms which tends to $\infty$ as $\varepsilon \rightarrow 0$, we define an operator $A_{e}^{R}$ as

$$
\left(A_{\varepsilon}^{R} v^{j}\right)(t)=\left(\frac{d^{2}}{d t^{2}}+\omega_{0}^{2}-i \frac{e^{2}}{6 \pi}\left|\frac{d}{d t}\right|^{3}\right) v^{v}(t) \text {. }
$$

So we get

$$
\begin{aligned}
& A_{\varepsilon}^{R} \frac{d}{d t} \frac{\delta Z}{\delta p_{j}(t)} \\
& =\frac{i}{h} \frac{d}{d t} p_{j}(t) Z-e \frac{i}{h}\left\langle\delta,\left\{\delta_{j k}+\Delta \square_{F^{\prime}}^{-1}\left(\delta_{j k}+\frac{\partial}{\partial x^{j}}(-\Delta)^{-1} \frac{\partial}{\partial x^{k}}\right)\right\} B^{k}(\cdot, t)\right\rangle Z .
\end{aligned}
$$

Combining this with (4.5), we have eliminated the 'variable' $B^{0}(x, t)$ in (II.1)(II.4).

In order to eliminate $\frac{d}{d t}$ in $(4.7)^{\prime}$, we proceed as follows: In (4.5) and $(4.7)^{\prime}$, there appears the operator $\delta_{j k}+\frac{\partial}{\partial x^{j}}(-\Delta)^{-1} \frac{\partial}{\partial x^{k}}$, which projects the vector to its divergence free part. So, it seems natural to assume that 'variable' $\boldsymbol{B}$ is divergence free, i.e. $\frac{\partial}{\partial x^{k}} B^{k}(x, t)=0$ for any $t$. In this case

$$
\left\{\delta_{j k}+\Delta \square F^{-1}\left(\delta_{j k}+\frac{\partial}{\partial x^{j}}(-\Delta)^{-1} \frac{\partial}{\partial x^{k}}\right)\right\} B^{k}=\frac{\partial^{2}}{\partial t^{2}} \square_{F}^{-1} B^{\jmath} .
$$

Inserting this into $(4.7)^{\prime}$, we get the following as the renormalized one of (II.1) with suitable constant $C^{3}$.

$$
\left(\frac{d^{2}}{d t^{2}}+\omega_{0}^{2}-i \frac{e^{2}}{6 \pi}\left|\frac{d}{d t}\right|^{3}\right) \frac{\delta Z}{\delta p_{j}(t)}=\frac{\imath}{h} p_{j}(t) Z-e \frac{\imath}{h}\left\langle\delta, \square \bar{F}^{1} \frac{\partial}{\partial t} B^{J}(\cdot, t)\right\rangle Z+C^{j}
$$

Here, we may put $C^{3}=0$ because $Z=0$ is a solution of (II. 1$)-$ (II.3) without $Z(0,0)$ $=1$.

So we get, for $\tilde{Z}=\tilde{Z}\left(\boldsymbol{p}, \boldsymbol{B}^{\prime}\right)$ for $\boldsymbol{B}^{\prime}(x, t)=\left(B^{1}(x, t), B^{2}(x, t), B^{3}(x, t)\right)$ independ ent of $B^{0}(x, t)$,

$$
\left(\frac{d^{2}}{d t^{2}}+\omega^{2}-2 \frac{e^{2}}{6 \pi}\left|\frac{d}{d t}\right|^{3}\right) \frac{\delta \tilde{Z}}{\delta p_{j}(t)}=\frac{l}{h} p_{j}(t) \tilde{Z}-e \frac{l}{h}\left\langle\delta, \Xi_{F^{1}} \frac{\partial}{\partial t} B^{2}(\cdot, t)\right\rangle \tilde{Z}
$$

and

$$
\square\left(\delta_{j k}+\frac{\partial}{\partial x^{k}}(-\Delta)^{-1} \frac{\partial}{\partial x^{k}}\right) \frac{\delta \tilde{Z}}{\delta B^{k}(x, t)}
$$




$$
=\frac{i}{h} B^{\prime}(x, t) \tilde{Z}-e\left(\delta_{j k}+\frac{\partial}{\partial x^{j}}(-\Delta)^{-1} \frac{\partial}{\partial x^{k}}\right) \delta(x) \frac{d}{d t} \frac{\delta \tilde{Z}}{\delta p_{k}(t)}
$$

with

$$
\tilde{Z}(0,0)=1 \text {. }
$$

Moreover, if the vector $\left\{\frac{\delta \tilde{Z}}{\delta B^{k}(x, t)}\right\}$ is assumed to be divergence free, then our problem are simplified in the following form: Find a functional $\tilde{Z}^{R}=$ $\widetilde{Z}^{R}\left(\boldsymbol{p}, \boldsymbol{B}^{\prime}\right)$ for $\boldsymbol{p}(t)=\left(p_{1}(t), p_{2}(t), p_{3}(t)\right)$ and $\boldsymbol{B}^{\prime}(x, t)=\left(B^{1}(x, t), B^{2}(x, t), B^{3}(x, t)\right)$ satisfying $\frac{\partial}{\partial x^{k}} B^{k}(x, t)=0$ such that

$$
\begin{aligned}
& \left(\frac{d^{2}}{d t^{2}}+\omega_{0}^{2}-i \frac{e^{2}}{6 \pi}\left|\frac{d}{d t}\right|^{3}\right) \frac{\delta \tilde{Z}^{R}}{\delta p_{j}(t)}=\frac{i}{h} p_{j}(t) \tilde{Z}^{R}-e \frac{i}{h}\left\langle\delta, \square \bar{F}^{-1} \frac{\partial}{\partial t} B^{\jmath}(\cdot, t)\right\rangle \tilde{Z}^{R}, \\
& \square \frac{\delta \widetilde{Z}^{R}}{\delta B^{\jmath}(x, t)}=\frac{i}{h} B^{\jmath}(x, t) \tilde{Z}^{R}-e\left\{\delta_{j k}+\frac{\partial}{\partial x^{j}}(-\Delta)^{-1} \frac{\partial}{\partial x^{k}}\right\} \delta(x)-\frac{d}{d t} \frac{\delta \widetilde{Z}^{R}}{\delta p_{k}(t)},
\end{aligned}
$$

$$
\frac{\partial}{\partial x^{k}} \frac{\delta \tilde{Z}^{R}}{\delta B^{k}(x, t)}=0
$$

and

$$
\tilde{Z}^{R}(0,0)=1
$$

Remark. (1) Here the procedures of renormalization and gauge fixing (in this case, this means the procedure of eliminating the 'variable' $B^{\circ}(x, t)$ and of assuming $\left.\frac{\partial}{\partial x^{k}} B^{k}(x, t)=0\right)$ occurs altogether.

(2) The condition ( $\Pi .3)^{\prime}$ is called Ward-Takahashi identity in physics literatures.

(3) If $Z\left(\boldsymbol{p}, \boldsymbol{B}^{\prime}\right)$ was known to be represented by the characteristic function of a certain measure $\mu\left(\boldsymbol{q}, \boldsymbol{A}^{\prime}\right)$ on the space of

$$
X=\left\{\left(\boldsymbol{q}(t), \boldsymbol{A}^{\prime}(x, t)\right): \frac{\partial}{\partial x^{k}} A_{k}(x, t)=0\right\}
$$

i.e.

$$
Z\left(\boldsymbol{p}, \boldsymbol{B}^{\prime}\right)=\int_{X} \exp -\frac{i}{h}\left(\langle\boldsymbol{q}, \boldsymbol{p}\rangle+\left\langle\boldsymbol{A}^{\prime}, \boldsymbol{B}^{\prime}\right\rangle\right) d \mu\left(\boldsymbol{q}, \boldsymbol{A}^{\prime}\right),
$$

then this equality is naturally satisfied by $Z$.

\section{§. Construction of an explicit solution of renormalized S-D equations $(\mathrm{II})$.}

In this section, we abbreviate prime and tilde in the problem (II). 
As in problem (I), we first consider the case where vector 'variable' $\boldsymbol{B}(x, t)$ satisfying $\left(\partial / \partial x^{k}\right) B^{k}(x, t)=0$, has its support with respect to $x$ in a ball $\Omega_{R}=$ $\left\{x \in R^{3}:|x|<R\right\}$.

In this case, our problem reduces to the following: Find a functional $Z_{\Omega}=$ $Z_{\Omega}(\boldsymbol{p}(\cdot), \boldsymbol{B}(\cdot, \cdot)), \boldsymbol{p}(t)=\left(p_{1}(t), p_{2}(t), p_{3}(t)\right), \boldsymbol{B}(x, t)=\left(B^{1}(x, t), B^{2}(x, t), B^{3}(x, t)\right)$, $\left(\partial / \partial x^{k}\right) B^{k}(x, t)=0$ and $\operatorname{supp} B^{\jmath}(\cdot, t) \subset \Omega_{R}$ satisfying

$$
\begin{gathered}
A_{e}^{R} \frac{\delta Z_{\Omega}}{\delta p_{j}(t)}=\frac{i}{h} p_{j}(t) Z_{\Omega}-e \frac{i}{h}\left\langle\delta, \square_{F}^{-1} \frac{\partial}{\partial t} B^{\jmath}(\cdot, t)\right\rangle Z_{\Omega}, \\
\square \frac{\delta Z_{\Omega}}{\delta B^{\jmath}(x, t)}=\frac{i}{h} B^{\nu}(x, t) Z_{\Omega}-e\left(\delta_{j k}+\frac{\partial}{\partial x^{j}}(-\Delta)^{-1} \frac{\partial}{\partial x^{k}}\right) \delta \frac{d}{d t} \frac{\delta Z_{\Omega}}{\delta p_{k}(t)}, \\
\frac{\partial}{\partial x^{k}} \frac{\delta Z_{\Omega}}{\delta \bar{B}^{k}(x, t)}=0
\end{gathered}
$$

and

$$
Z_{\Omega}(0,0)=1 \text {. }
$$
$1,2, \cdots$,

Let $\left\{\mu_{l}^{2}\right\},\left\{\boldsymbol{w}_{l}(x)\right\}$ be eigenvalues and eigenvectors satisfying $\jmath=1,2,3, l=$

$$
\begin{gathered}
-\Delta w_{l}^{3}(x)+\frac{\partial}{\partial x^{3}} \pi_{l}(x)=\mu_{l}^{2} w_{l}^{3}(x) \text { in } \Omega_{R}, \\
\frac{\partial}{\partial x^{j}} w_{l}^{3}(x)=0
\end{gathered}
$$

and

$$
\left.w_{l}^{3}(x)\right|_{\partial \Omega_{R}}=0 .
$$

Here, $\boldsymbol{w}_{l}(x) \cdot \boldsymbol{w}_{k}(y)=\sum_{j=1}^{3} w_{l}^{\jmath}(x) w_{k}^{j_{l}}(y)$ and $\boldsymbol{w}_{l}(x)^{\prime}$ s are normalized as $\int_{\Omega_{R}} w_{l}^{2}(x) w_{k}^{\jmath}(x) d x$ $=(1 / 3) \delta_{l k} \delta_{i j}$. (We assume as if $\boldsymbol{w}_{l}(\cdot)$ 's are taken to be symmetric in all directions in $\boldsymbol{R}^{3}$.)

If 'variable' $\boldsymbol{B}(x, t)$ satisfies $\frac{\partial}{\partial x^{k}} B^{k}(x, t)=0$ and supp $B^{\prime}(\cdot, t) \subset \Omega_{R}$, we may expand $\boldsymbol{B}(x, t)$ as $B^{\jmath}(\cdot, t)=\sum_{l=1}^{\infty} b_{l}(t) w_{t}^{\prime}(\cdot)$ where $b_{l}(t)=\int_{\Omega_{R}} \boldsymbol{B}(x, t) \cdot \boldsymbol{w}_{l}(x) d x$. So we may identify $\boldsymbol{B}(\cdot, t)$ with $\left\{b_{l}(t)\right\}$. (Be aware that $B^{\prime}(\cdot, t)$ is assumed to be identified with $\left\{b_{l}(t)\right\}$ independent of $j$.) Moreover, we assume, for the time being, $p_{j}(t)$ is $j$ independent.

Putting $\sigma_{l}^{3}=\left\langle\delta, w_{l}^{3}(\cdot)\right\rangle=\sigma_{l}$, we reformulate the above problem as follows: Find a functional $Z=Z\left(\boldsymbol{p},\left\{b_{l}\right\}\right)$ such that

$$
\begin{gathered}
A_{e}^{R} \frac{\delta Z}{\delta p_{j}(t)}=\frac{i}{h} p_{j}(t) Z-e \frac{i}{3 h} \sigma_{l} C_{l, F}^{-1} \frac{d}{d t} \frac{\delta Z}{\delta b_{l}(t)}, \\
C_{l} \frac{\delta Z}{\delta b_{l}(t)}=\frac{i}{h} b_{l}(t) Z-e \sigma^{3} \frac{d}{d t} \frac{\delta Z}{\delta p_{j}(t)}
\end{gathered}
$$


and

$$
Z(0,0)=1
$$

Here $C_{l}=\frac{d^{2}}{d t^{2}}+\mu_{l}^{2}$ and

$$
C_{l . F}^{-1}=\frac{1}{2 \mu_{l}}\left(\theta(t) e^{-\imath \mu_{l} t}+\theta(-t) e^{\imath \mu_{l} t}\right) .
$$

As before, the kernel of the inverse operator of $\square$ in $\Omega_{R}$ is given

$$
G_{F, \Omega}^{e}(x, y, t)=\sum_{l=1}^{\infty} \frac{\boldsymbol{w}_{l}(x) \cdot \boldsymbol{w}_{l}(y)}{2 \mu_{l}}\left(\theta(t) e^{-\imath \mu_{l} t}+\theta(-t) e^{\imath \mu_{l} t}\right) .
$$

Instead of above problems, we consider the following first order partial differential operators with countable variables:

$$
\begin{gathered}
a_{e} u_{x}=\frac{i}{h} x u-\frac{i}{3 h} \tilde{e} \sigma_{l} c_{l}^{-1} y^{l} u, \\
c_{l} u_{y} l=\frac{i}{h} y^{l} u-3 \tilde{e} \sigma_{l} u_{x}
\end{gathered}
$$

and

$$
u(0,0)=1
$$

where $\tilde{e}$ corresponds to $e \frac{d}{d t}$. A solution of this system is given by

$$
\begin{aligned}
u(x, y) & =\exp \left[\frac{i}{2 h} a_{e}^{-1} x^{2}+\frac{i}{2 h} \sum_{l=1}^{\infty}\left(\frac{y^{l}}{c_{l}}\right)^{2}\right. \\
& \left.+\frac{i}{2 h} \tilde{e}^{2} \frac{1}{3 a_{e}}\left(\sum_{l=1}^{\infty} \frac{\sigma_{l} y^{l}}{c^{l}}\right)^{2}-\frac{i}{h} \tilde{e} \frac{1}{3 a_{e}} x \sum_{l=1}^{\infty}\left(\frac{\sigma_{l} y^{l}}{c_{l}}\right)\right] .
\end{aligned}
$$

From this expression, we may put as

$$
\begin{aligned}
& Z^{R}(\boldsymbol{p}, \boldsymbol{B})=\exp \left[\frac{i}{2 h}\left\langle\left(A_{e}^{R}\right)^{-1} p_{\jmath}, p_{\jmath}\right\rangle+\frac{\imath}{2 h}\left\langle\square_{F}^{-1} B^{\jmath}, B^{\jmath}\right\rangle\right. \\
& \left.+e^{2} \frac{i}{2 h}\left\langle\left(A_{e}^{R}\right)^{-1}\left\langle\delta, \square_{F}^{-1} \frac{\partial}{\partial t} B^{\jmath}\right\rangle,\left\langle\delta, \square_{F}^{-1} \frac{\partial}{\partial t} B^{j}\right\rangle\right\rangle-e \frac{\imath}{h}\left\langle\left(A_{e}^{R}\right)^{-1} p_{\jmath},\left\langle\delta, \square_{\bar{F}}^{-1} \frac{\partial}{\partial t} B^{j}\right\rangle\right\rangle\right]
\end{aligned}
$$

Or, we may define

$$
\begin{aligned}
\tilde{Z}^{R}(\boldsymbol{p}, \boldsymbol{B})= & \exp \left[\frac{i}{2 h}\left\langle\left(A_{\varepsilon}^{R}\right)^{-1} p_{\jmath}, p_{\jmath}\right\rangle+\frac{\imath}{2 h}\left\langle\square_{F}^{-1} P_{j k} B^{k}, B^{\jmath}\right\rangle\right. \\
& +e^{2} \frac{i}{2 h}\left\langle\left(A_{e}^{R}\right)^{-1}\left\langle\delta, \square \bar{F}^{-1} P_{j k} \frac{\partial}{\partial t} B^{k}\right\rangle,\left\langle\delta, \square \square_{\bar{F}}^{-1} P_{j k} \frac{\partial}{\partial t} B^{k}\right\rangle\right\rangle \\
& \left.-e \frac{i}{h}\left\langle\left(A_{e}^{R}\right)^{-1} p_{\jmath},\left\langle\delta, \square \bar{F}^{-1} P_{j k} \frac{\partial}{\partial t} B^{k}\right\rangle\right\rangle\right]
\end{aligned}
$$


where $P_{j k}=\delta_{j k}+\frac{\partial}{\partial x^{j}}(-\Delta)^{-1} \frac{\partial}{\partial x^{k}}$.

THEOREM II. A functional $Z^{R}$ defined above is well-defined on the space

$$
\left\{(\boldsymbol{p}, \boldsymbol{B}) \in H^{-1}(\boldsymbol{R}) \times\left(C_{0}^{\infty}\left(\boldsymbol{R}^{4}\right)\right)^{3} ; \frac{\partial}{\partial x^{k}} B^{k}(x, t)=0\right\}
$$

and infintely differentiable. Moreover, it satisfies (II.1) ${ }_{R, g}^{\prime},(\mathrm{II} .2)^{\prime},(\mathrm{II} .3)^{\prime}$ and (II.4).

Remark. (1) If we consider $\tilde{Z}^{R}$ instead of $Z^{R}$, then we define the functional on the spate where $\boldsymbol{B}$ does not necessarily satisfy $\frac{\partial}{\partial x^{k}} B^{k}(x, t)=0$.

(2) Assuming the symmetry in $\left\{\boldsymbol{w}_{l}(\cdot)\right\}$, we get the result (5.15) or (5.16). So, at least formally, there exists another possibility of the non-uniqueness of the solution of (II), but we conjecture that this solution is essentially unique.

(3) Calculations of Green functions of this problem is omitted here.

Appendix A. Derivations of formal S-D equations.

(I) Let a Lagrangean $L(q, v)$ be given as

$$
\begin{aligned}
L(q, v)= & \frac{1}{2} \int_{R}\left(\dot{q}(t)^{2}-\omega_{0}^{2} q(t)^{2}\right) d t \\
& +\frac{1}{2} \int_{R^{4}}\left(\left|\frac{\partial}{\partial t} v(x, t)\right|^{2}-|\nabla v(x, t)|^{2}\right) d x d t-\lambda \int_{R^{4}} \delta(x) q(t) v(x, t) d x d t
\end{aligned}
$$

where $\nabla v(x, t)=\left(\frac{\partial}{\partial x^{1}} v(x, t), \frac{\partial}{\partial x^{2}} v(x, t), \frac{\partial}{\partial x^{3}} v(x, t)\right)$ and $\dot{q}(t)=\frac{d}{d t} q(t)$.

Following Feynman's idea, we quantize the dynamics governed by $L(q, v)$, that is, we consider the functional

$$
Z(p, u)=\frac{\int \exp \frac{i}{h} L(q, v) \cdot \exp -\frac{l}{h}(\langle q, p\rangle+\langle v, u\rangle) d_{F} q d_{F} v}{\int \exp \frac{i}{h} L(q, v) d_{F} q d_{F} v}
$$

where $h$ is Planck's constant divided by $2 \pi, d_{F} q$ and $d_{F} v$ are Feynman measures on the spaces $\{q(t) ; t \in \boldsymbol{R}\}$ and $\left\{v(x, t) ;(x, t) \in \boldsymbol{R}^{4}\right\}$, respectively. Here, we assume that functions $q(t), v(x, t)$ and $p(t), u(x, t)$ are real valued. $(p(t)$ and $u(x, t)$ are called external sources.)

$Z(p, u)$ is considered as the characteristic functional of the 'complex canonical ensemble' $\exp \frac{i}{h} L(q, v) d_{F} q d_{F} v$ with the normalizing constant.

Though there exists no Feynman measure mathematically (R. H. Cameron [5]), we may derive (I.1) and (I.2) from (A.2) assuming as if it exists. 
Putting $\hat{Z}=\hat{Z}(q, v)=\exp \frac{i}{h} L(q, v)$, we have

$$
\frac{\delta \hat{Z}}{\delta q(t)}=-\frac{i}{h}\left(\frac{d^{2}}{d t^{2}}+\omega_{0}^{2}\right) q(t) \hat{Z}-\frac{i}{h} \lambda \int_{R^{3}} \delta(x) v(x, t) d x \hat{Z}
$$

and

$$
\frac{\delta \hat{Z}}{\delta v(x, t)}=-\frac{i}{h} \square \hat{Z}-\frac{i}{h} \lambda \delta(x) q(t) \hat{Z} .
$$

Assuming that the integration by parts holds, we have

$$
\frac{\int \frac{\delta \hat{Z}}{\delta q(t)} \exp -\frac{i}{h}(\langle q, p\rangle+\langle v, u\rangle) d_{F} q d_{F} v}{\int \hat{Z} d_{F} q d_{F} v}=\frac{i}{h} p(t) Z(p, u)
$$

and

$$
\frac{\int \frac{\delta \hat{Z}}{\delta v(x, t)} \exp -\frac{i}{h}(\langle q, p\rangle+\langle v, u\rangle) d_{F} q d_{F} v}{\int \hat{Z} d_{F} q d_{F} v}=\frac{i}{h} u(x, t) Z(p, u)
$$

On the other hand, interchanging formally the integration and the differentiation, we get

$$
\frac{\delta Z}{\delta p(t)}=\frac{-\frac{i}{h} \int q(t) \hat{Z}(q, v) \exp -\frac{i}{h}(\langle q, p\rangle+\langle v, u\rangle) d_{F} q d_{F} v}{\int \hat{Z} d_{F} q d_{F} v}
$$

and

$$
\frac{\delta Z}{\delta u(x, t)}=-\frac{i j \int v(x, t) \hat{Z}(q, v) \exp -\frac{i}{h}(\langle q, p\rangle+\langle v, u\rangle) d_{r} q d_{F} v}{\int \hat{Z} d_{F} q d_{F} v}
$$

Inserting (A.3) into (A.5) and using (A.7) and (A.8), we get readily (I.1). Analogously (I.2) is obtained.

The equations governing the classical mechanics corresponding to (A.1) are given by

$$
\begin{gathered}
\left(\frac{d^{2}}{d t^{2}}+\omega_{0}^{3}\right) q(t)=-\lambda \int_{R^{3}} \delta(x) v(x, t) d x, \\
\square v(x, t)=-\lambda \delta(x) q(t) .
\end{gathered}
$$

These equations are first introduced by F. Schwahbl \& W. Thirring [17] and the classical initial value problem is studied by P. C. Aichelburg \& R. Beig [1]. On the other hand, the quantization of this system by using the Fock space representation after smearing $\delta(x)$, that is, replacing $\delta(x)$ by $\rho_{\hat{\varepsilon}}(x)$ is given mathematically by A. Arai [3]. 
(II) In this case, we consider a Lagrangean $L(\boldsymbol{q}, \boldsymbol{A})$ given by

$$
\begin{aligned}
& L(\boldsymbol{q}, \boldsymbol{A})=\frac{1}{2} \int_{R}\left(m^{2} \dot{q}^{\jmath}(t)^{2}-\omega_{0}^{2} q^{\jmath}(t)^{2}\right) d t \\
& \quad+\frac{1}{2} \int_{R^{4}}\left[\left\{\frac{\partial}{\partial x^{3}} A_{0}(x, t)-\frac{\partial}{\partial t} A_{j}(x, t)\right\}^{2}-\frac{1}{2}\left\{\frac{\partial}{\partial x^{j}} A_{2}(x, t)-\frac{\partial}{\partial x^{2}} A_{j}(x, t)\right\}^{2}\right] d x d t \\
& \quad+e \int_{\boldsymbol{R}^{4}} \delta(x) q^{\jmath}(t)\left\{\frac{\partial}{\partial t} A_{J}(x, t)-\frac{\partial}{\partial x^{j}} A_{0}(x, t)\right\} d x d t .
\end{aligned}
$$

Corresponding physical studies are made by R. E. Norton \& W. K. R. Watson [15], and K. Rzazewski \& W. Zakowicz [16].

As before, $Z(\boldsymbol{p}, \boldsymbol{B})$ is defined formally as

$$
Z(\boldsymbol{p}, \boldsymbol{B})=\frac{\int \exp \frac{i}{h} L(\boldsymbol{q}, \boldsymbol{A}) \cdot \exp -\frac{i}{h}(\langle\boldsymbol{q}, \boldsymbol{p}\rangle+\langle\boldsymbol{A}, \boldsymbol{B}\rangle) d_{F} \boldsymbol{q} d_{F} \boldsymbol{A}}{\int \exp \frac{i}{h} L(\boldsymbol{q}, \boldsymbol{A}) d_{F} \boldsymbol{q} d_{F} \boldsymbol{A}}
$$

where $\boldsymbol{q}=\left(q^{1}, q^{2}, q^{3}\right)$ and $\boldsymbol{A}=\left(A_{0}, A_{1}, A_{2}, A_{3}\right)$. Then, we get (II.1)-(II.4) after the same procedure as we derive (I.1)-(I.3) from (A.2).

The equations governing the classical mechanics corresponding to (A.11) are

$$
\begin{aligned}
& m^{2} \ddot{q}^{\jmath}(t)+\omega_{0}^{2} q^{\jmath}(t)=e \int_{R^{3}} \delta(x)\left\{\frac{\partial}{\partial t} A_{j}(x, t)-\frac{\partial}{\partial x^{j}} A_{0}(x, t)\right\} d x, \\
& \square A_{j}(x, t)+\frac{\partial}{\partial x^{j}} \frac{\partial}{\partial x^{k}} A_{k}(x, t)-\frac{\partial}{\partial x^{\jmath}} \frac{\partial}{\partial t} A_{0}(x, t)=-e \delta(x) \dot{q}^{\jmath}(t)
\end{aligned}
$$

and

$$
\Delta A_{0}(x, t)-\frac{\partial}{\partial x^{k}} \frac{\partial}{\partial t} A_{k}(x, t)=e \frac{\partial}{\partial x^{j}} \delta(x) q^{\jmath}(t) .
$$

Eliminating $A_{0}(x, t)$ from above equations, we have

$$
\begin{aligned}
& m^{2} \ddot{q}^{\jmath}(t)+\left(\omega_{0}^{2}+\frac{e^{2}}{3} \int_{R^{3}}|\delta(x)|^{2} d x\right) q^{\jmath}(t) \\
& =e \int_{R^{3}} \delta(x)\left(\delta_{j k}+\frac{\partial}{\partial x^{j}}(-\Delta)^{-1} \frac{\partial}{\partial x^{k}}\right) \frac{\partial}{\partial t} A_{k}(x, t) d x
\end{aligned}
$$

and

$$
\square\left(\delta_{j k}+\frac{\partial}{\partial x^{j}}(-\Delta)^{-1} \frac{\partial}{\partial x^{k}}\right) A_{k}(x, t)=e\left(\delta_{j k}+\frac{\partial}{\partial x^{j}}(-\Delta)^{-1} \frac{\partial}{\partial x^{k}}\right)\left(\delta(x) q^{k}(t)\right) .
$$

As before, we treated $\delta(x)$ as if it is a function.

On the other hand, we may derive the equations (A.16) and (A.17) from the following Lagrangean.

$$
\widetilde{L}\left(\boldsymbol{q}, \boldsymbol{A}^{\prime}\right)=\frac{1}{2} \int_{R}\left(m^{2} \dot{q}^{\jmath}(t)^{2}-\boldsymbol{\omega}^{2} q^{\jmath}(t)^{2}\right) d t
$$




$$
\begin{aligned}
& +\frac{1}{2} \int_{R^{4}}\left[\left\{\left(\delta_{j k}+\frac{\partial}{\partial x^{j}}(-\Delta)^{-1} \frac{\partial}{\partial x^{k}}\right) \frac{\partial}{\partial t} A_{k}(x, t)\right\}^{2}\right. \\
& \left.-\frac{1}{2}\left\{\frac{\partial}{\partial x^{j}} A_{i}(x, t)-\frac{\partial}{\partial x^{2}} A_{j}(x, t)\right\}^{2}\right] d x d t \\
& +e \int_{R^{4}} \delta^{4}(x) q^{j}(t)\left\{\left(\delta_{j k}+\frac{\partial}{\partial x^{j}}(-\Delta)^{-1} \frac{\partial}{\partial x^{k}}\right) \frac{\partial}{\partial t} A_{k}(x, t)\right\} d x d t
\end{aligned}
$$

where $\boldsymbol{A}^{\prime}=\left(A_{1}, A_{2}, A_{3}\right)$. Here $\boldsymbol{\omega}$ is given formally as

$$
\omega=\omega_{0}^{2}+\frac{e^{2}}{3} \int_{R^{3}}|\delta(x)|^{2} d x .
$$

For such $\tilde{L}\left(\boldsymbol{q}, \boldsymbol{A}^{\prime}\right)$, we define $\tilde{Z}=\tilde{Z}\left(\boldsymbol{p}, \boldsymbol{B}^{\prime}\right)$ as same as before. Then, we get

$$
\begin{aligned}
& \left(m^{2} \frac{d^{2}}{d t^{2}}+\omega^{2}\right) \frac{\delta \tilde{Z}}{\delta p_{j}(t)} \\
& =\frac{i}{h} p_{j}(t) \tilde{Z}+e \int_{R^{3}}\left(\delta_{j k}+\frac{\partial}{\partial x^{j}}(-\Delta)^{-1}-\frac{\partial}{\partial x^{k}}\right) \delta(x) \frac{\partial}{\partial t} \frac{\delta \tilde{Z}}{\delta B^{k}(x, t)} d x, \\
& \square\left(\delta_{j k}+\frac{\partial}{\partial x^{j}}(-\Delta)^{-1} \frac{\partial}{\partial x^{k}}\right) \frac{\delta \tilde{Z}}{\delta B^{k}(x, t)} \\
& =\frac{i}{h} B^{j}(x, t) \tilde{Z}-e\left(\delta_{j k}+\frac{\partial}{\partial x^{j}}(-\Delta)^{-1} \frac{\partial}{\partial x^{k}}\right) \delta(x) \frac{d}{d t} \frac{\delta \tilde{Z}}{\delta p_{k}(t)}
\end{aligned}
$$

After eliminating the product of distributions in (A.20) as same as in $\$ 4$, we may say that $\widetilde{Z}$ also satisfies the equations (4.12)-(4.13). So, in this case, using the information obtained by eliminating $A_{0}(x, t)$ from (A.13)-(A.15), we get the same quantized version.

Remark. (1) In spite of the above calculations, "quantization after eliminating $A_{0}$ ' and 'eliminating $B^{0}$ after quantization' must be distinguished in general.

(2) The reason why the condition (4.3) is naturally satisfied in problem (II) is explained as follows :

As the Lagrangean $L(\boldsymbol{q}, \boldsymbol{A})$ is gauge invariant, i. e.

$$
L(\boldsymbol{q}, \boldsymbol{A})=L(\boldsymbol{q}, \boldsymbol{A}+\tilde{\nabla} \varphi) \quad \text { for any } \varphi \in C_{0}^{\infty}\left(\boldsymbol{R}^{4}\right)
$$

where $\tilde{\nabla} \varphi=\left\langle\partial_{t} \varphi, \nabla \varphi\right)$, combining this with the translational invariance of the Feynman measure, we get

$$
Z(\boldsymbol{p}, \boldsymbol{B})=\exp \frac{i}{h}\langle\boldsymbol{B}, \tilde{\nabla} \varphi\rangle Z(\boldsymbol{p}, \boldsymbol{B}) \quad \text { for any } \varphi \text {. }
$$

This equation is equivalent to (4.3). 
Appendix B. The Feynman propagator.

To derive renormalized S-D equations in $\S 3$ and $\S 5$, it is necessary to consider the following division problems in $D^{\prime}\left(\boldsymbol{R}^{n}\right)$.

$$
\Delta E(x)=\delta(x) \quad \text { in } \quad D^{\prime}\left(\boldsymbol{R}^{3}\right)
$$

and

$$
\square E(x, t)=\delta(x, t) \quad \text { in } \quad D^{\prime}\left(\boldsymbol{R}^{4}\right) .
$$

The solution of (B.1) is rather well-known and it is given by

$$
E(x)=(2 \pi)^{-3} \int_{R^{3}} \frac{e^{2 x \xi}}{|\xi|^{2}} d \xi=\frac{1}{4 \pi|x|} .
$$

On the other hand, we define

$$
E(x, t)=\lim _{\varepsilon \rightarrow 0}(2 \pi)^{-4} \int_{R^{4}} \frac{e^{-\imath t \tau+2 x \xi}}{-\tau^{2}+|\xi|^{2}-i \varepsilon} d \tau d \xi .
$$

This is the definition of the distribution $G_{F}^{B}(x, t)$. (In I. M. Gelfand \& G. E. Shilov [10], a slightly different definition is given.)

Remark. In defining (B.4), we use $-i \varepsilon$ to regularize the integral. But, mathematically, there exists no preference to use $-i \varepsilon$ or $+i \varepsilon$.

Moreover, using the polar coordinates, we have another expression.

$$
G_{F}^{B}(x, t)=\frac{i}{8 \pi^{2}}\left\{p \cdot v \cdot \frac{1}{|x|-t}+p \cdot v \cdot \frac{1}{|x|+t}-i \pi(\delta(|x|-t)+\delta(|x|+t))\right\} .
$$

Here, p.v. means to take the principal value. In fact, by putting

$$
\hat{G}_{\varepsilon}(x, \tau)=(2 \pi)^{-3} \int_{R^{3}} \frac{e^{2 x \xi}}{-\tau^{2}+|\xi|^{2}-\imath \varepsilon} d \xi .
$$

Using the polar coordinates and without bothering the interchange of integral, we get readily

$$
\hat{G}_{s}(\tau, x)=\frac{\exp i|x| \sqrt{\tau^{2}+i \varepsilon}}{4 \pi|x|}
$$

Here, the branch of the square root is taken so that the imaginary part is positive.

Making $\varepsilon$ tends to 0 , we get

$$
\frac{1}{\sqrt{ } 2 \pi} \int_{R} G_{F}^{B}(x, t) e^{i t \tau} d t=\frac{e^{i|\tau||x|}}{4 \pi|x|} .
$$

Applying the Fourier inverse transform in $\tau$, we get (B.5). Putting the right hand side of above equation by $\hat{G}(x, \tau)$, we define the following operator. 


$$
(\hat{G} f)(\tau)=\int_{R^{3}} \hat{G}(x, \tau) f(x, \tau) d x .
$$

Then, for $N>1$ and any $m$, we get

$$
\left(1+\tau^{2}\right)^{m}|(\hat{G} f)(\tau)| \leqq \text { const. } \sup \left[\left(1+\tau^{2}\right)^{m}\left(1+|x|^{2}\right)^{N}|f(\tau, x)|\right]
$$

From this, we obtain the proof of Lemma 3.1.

Remark. This suggests that we may extend Lemma 3.1, to more broader space than $C_{0}^{\infty}\left(\boldsymbol{R}^{4}\right)$. But this point is not considered further here.

Appendix C. Generalizations.

We may extend our results to the following cases.

(1) Instead of (A.1), we may consider

$$
\begin{aligned}
L^{N}\left(\boldsymbol{q}, v ;\left\{\boldsymbol{a}_{\mu}\right\}\right)= & \frac{1}{2} \int_{R}\left(\dot{q}_{\mu}^{2}(t)-\omega_{\mu}^{2} q_{\mu}^{2}(t)\right) d t \\
& +\frac{1}{2} \int_{R^{4}}\left(\left|\partial_{t} v(x, t)\right|^{2}-|\nabla v(x, t)|^{2}\right) d x d t \\
& -\lambda \int_{R^{4}} \delta\left(x-\boldsymbol{a}_{\mu}\right) q_{\mu}(t) v(x, t) d x d t
\end{aligned}
$$

where $\left\{\boldsymbol{a}_{\mu}\right\}$ are $N$ points in $\boldsymbol{R}^{3}$.

The equations corresponding to the classical mechanics of (C.1) are

$$
\begin{gathered}
\ddot{q}_{\mu}(t)+\omega_{\mu}^{2} q_{\mu}(t)^{2}=-\lambda \int_{R^{3}} \delta\left(x-\boldsymbol{a}_{\mu}\right) v(x, t) d x, \\
\square v(x, t)=-\lambda \delta\left(x-\boldsymbol{a}_{\nu}\right) q_{\nu}(t) .
\end{gathered}
$$

An initial value problem of these equations for the case $N=2$ and $\omega_{1}==\omega_{2}$ is studied in P.C. Aichelburg \& H. Grosse [2]. The quantized case for $N$ arbitrary and $\boldsymbol{a}_{\mu}=0$ with $\delta(x)$ replaced by $\rho_{\varepsilon}(x)$ is treated in A. Inoue [12].

Defining $Z=Z\left(\boldsymbol{p}, u ;\left\{\boldsymbol{a}_{n}\right\}\right)$ for (C.1), we have

$$
\begin{gathered}
\left(\frac{d^{2}}{d t^{2}}+\omega_{\mu}^{2}\right) \frac{\delta Z}{\delta p_{\mu}(t)}=\frac{i}{h} p_{\mu}(t) Z-\lambda \int_{R^{3}} \delta\left(x-\boldsymbol{a}_{\mu}\right) \frac{\delta Z}{\delta u(x, t)} d x, \\
\square \frac{\delta Z}{\delta u(x, t)}=\frac{\imath}{h} u(x, t) Z-\lambda \delta\left(x-\boldsymbol{a}_{2}\right) \frac{\delta Z}{\delta p_{2}(t)}
\end{gathered}
$$

and

$$
Z(0,0)=1
$$

In this case, the renormalized operator $A_{k, x}^{R}$ becomes a $N \times N$ matrix operator with 


$$
\begin{aligned}
\left(A_{\lambda, N}^{R} \widehat{\boldsymbol{v}}\right)_{\mu}(\tau)= & \delta_{\mu \nu}\left(-\tau^{2}+\omega_{\nu}^{2}-i \frac{\lambda^{2}}{4 \pi}|\tau|\right) \hat{v}_{\nu}(\tau) \\
& -\lambda^{2} \sum_{\mu \neq \nu} \frac{1}{4 \pi\left|\boldsymbol{a}_{\mu}-\boldsymbol{a}_{\nu}\right|} e^{2 \mid a_{\mu}-a_{\nu|| \tau \mid} \hat{v}_{\nu}(\tau)}
\end{aligned}
$$

In other word,

$$
\begin{aligned}
\left(A_{\lambda, N}^{R} \boldsymbol{v}\right)_{\mu}(t)= & \delta_{\mu \nu}\left(\frac{d^{2}}{d t^{2}}+\omega_{\nu}^{2}-\imath \frac{\lambda^{2}}{4 \pi}\left|\frac{d}{d t}\right|\right) v_{\nu}(t) \\
-\lambda^{2} \sum_{\mu \neq \nu} & \frac{i}{8 \pi^{2}\left|\boldsymbol{a}_{\mu}-\boldsymbol{a}_{\nu}\right|} \int_{R}\left\{p \cdot v \cdot \frac{1}{t-s-\left|\boldsymbol{a}_{\mu}-\boldsymbol{a}_{\nu}\right|}+p \cdot v \cdot \frac{1}{t-s+\left|\boldsymbol{a}_{\mu}-\boldsymbol{a}_{\nu}\right|}\right. \\
& \left.\quad-i \pi\left(\delta\left(t-s-\left|\boldsymbol{a}_{\mu}-\boldsymbol{a}_{\nu}\right|\right)+\delta\left(t-s+\left|\boldsymbol{a}_{\mu}-\boldsymbol{a}_{\nu}\right|\right)\right)\right\} v(s) d s \quad(\mathrm{C} .7)^{\prime}
\end{aligned}
$$

Here, we interprete the last term of the above equation as equal to

$$
-\imath \frac{\lambda^{2}}{4 \pi}\left|\frac{d}{d t}\right| v_{\nu}(t) \quad \text { if } \quad\left|\boldsymbol{a}_{\mu}-\boldsymbol{a}_{\nu}\right|=0
$$

By the same argument as before, we get

$$
\begin{aligned}
Z\left(\boldsymbol{p}, u ;\left\{\boldsymbol{a}_{\mu}\right\}\right)= & \exp \left[\frac{i}{2 h}\left\langle\left(A_{\lambda, N}^{R}\right)^{-1} \boldsymbol{p}, \boldsymbol{p}\right\rangle+\frac{\imath}{2 h}\left\langle\square_{F}^{-1} u, u\right\rangle\right. \\
& +\frac{i}{2 h} \lambda^{2}\left\langle\left(A_{\lambda, N}^{R}\right)^{-1}\left\langle\delta\left(\cdot-\boldsymbol{a}_{v}\right), \square_{F}^{-1} u\right\rangle,\left\langle\delta\left(\cdot-\boldsymbol{a}_{\mu}\right), \square_{F}^{-1} u\right\rangle\right\rangle \\
& \left.-\frac{i}{h} \lambda\left\langle\left(\left(A_{\lambda, N}^{R}\right)^{-1} \boldsymbol{p}\right)_{\mu^{\prime}}\left\langle\delta\left(\cdot-\boldsymbol{a}_{\mu}\right), \square_{F}^{-1} u\right\rangle\right\rangle\right]
\end{aligned}
$$

where $\left(A_{\lambda, N}^{R}\right)^{-1}$ is the inverse operator of $A_{\lambda, N}^{R}$ when it exists.

(2) In [20], Zachariasen proposed to quantize the following equations.

$$
\begin{gathered}
\left(\square+m^{2}\right) u(x, t)=-\lambda \int_{R} f(s) w(x, t, s) d s, \\
(\square+s) w(x, t, s)=-\lambda f(s) u(x, t)
\end{gathered}
$$

where $f(s)=\frac{1}{4 \pi}\left(\frac{s-4 \mu_{0}^{2}}{s}\right)^{1 / 4} \theta\left(s-4 \mu_{0}^{2}\right)$.

These equations are derived from the following Lagrangean.

$$
\begin{aligned}
L(u, w)= & \frac{1}{2} \int_{R^{4}}\left(\left|\partial_{t} u(x, t)\right|^{2}-|\nabla u(x, t)|^{2}-m^{2} u(x, t)^{2}\right) d x d t \\
& +\frac{1}{2} \int_{R^{5}}\left(\left|\partial_{t} w(x, t, s)\right|^{2}-|\nabla w(x, t, s)|^{2}-s w(x, t, s)^{2}\right) d x d t d s \\
& -\lambda \int_{R^{5}} f(s) u(x, t) w(x, t, s) d x d t d s
\end{aligned}
$$


Defining $Z=Z(\varphi, \phi)$ as before, we have the following equations.

$$
\begin{aligned}
& (\square+m)^{2} \frac{\delta Z}{\delta \varphi(x, t)}=\frac{i}{h} \varphi(x, t) Z-\lambda \int_{R} f(s) \frac{\delta Z}{\delta \phi(x, t, s)} d s, \\
& (\square+s) \frac{\delta Z}{\delta \phi(x, t, s)}=\frac{i}{h} \phi(x, t, s) Z-\lambda f(s) \frac{\delta Z}{\delta \varphi(x, t)}
\end{aligned}
$$

and

$$
Z(0,0)=1
$$

The structure of these equations are same as those of (I.1)-(I.3) except $\delta(x)$ is replaced by $f(s)$. Here $f(s)$ is a function but is not infinitely differentiable, so it is singular when it is multiplied to distributions.

In this case, instead of $A^{\infty}$ in $\S 2$, we consider

$$
\square{ }^{\infty} u(x, t)=\left(\square+m^{2}\right) u(x, t)-\lambda^{2}\left\langle f(s),(\square+s)_{F}^{-1} f(s) u(x, t)\right\rangle .
$$

Using the Fourier transform with respect to $(x, t)$, we get

$$
\left(\square^{\infty} \hat{u}\right)(\xi, \tau)=\left(-\tau^{2}+|\xi|^{2}+m^{2}\right) \hat{u}(\xi, \tau)-\lambda^{2} \int_{R} \frac{|f(s)|^{2} \hat{u}(\xi, \tau)}{-\tau^{2}+|\xi|^{2}+s-i 0} d s .
$$

As

$$
\frac{1}{-\tau^{2}+|\xi|^{2}+s-i 0}-\frac{1}{s}=\frac{\tau^{2}-|\xi|^{2}}{s\left(-\tau^{2}+|\xi|^{2}+s-i 0\right)}
$$

and the last term in the above equation is integrable in $s$ at infinity, we define

$$
\square \square_{\lambda}^{R} u=\left(\square+m^{2}\right) u-\lambda^{2} \square \int_{R}(\square+s)_{\vec{F}} \frac{f(s)}{s} u d s .
$$

Then, we have

$$
\begin{aligned}
Z(\varphi, \phi)= & \exp \left[\frac{i}{2 h}\left\langle(\square)^{R}\right)_{F}^{-1} \varphi, \varphi\right\rangle+\frac{\imath}{2 h}\left\langle(\square+s)_{F}^{-1} \psi, \phi\right\rangle \\
& +\frac{i}{2 h} \lambda^{2}\left\langle\left(\square{ }_{\lambda}^{R}\right)_{F}^{-1}\left\langle f,(\square+s)_{F}^{-1} \psi\right\rangle,\left\langle f,(\square+s)_{F}^{-1} \psi\right\rangle\right\rangle \\
& \left.-\frac{i}{h} \lambda\left\langle\left(\square \square_{\lambda}^{R}\right)_{F}^{-1} \varphi,\left\langle f,(\square+s)_{F}^{-1} \psi\right\rangle\right\rangle\right] .
\end{aligned}
$$

(3) Same considerations may be work on the study of non-relativistic QED with the Schrödinger field as an exterior field. But this model will be studied in the forth comming paper.

Acknowledgements. The author expresses his hearty thanks to Dr. A. Arai whose knowledge of the Q.F.T. helps him very much, especially he brings the equations (A.9)-(A.10) and (A.16)-(A.17) with references to his attention. 


\section{REFERENCES}

[1] P.C. Aichelburg and R. Beig, Radiation damping as an initial value problem, Ann. of Phy., 98 (1976), 264-283.

[2] P.C. Aichelburg and H. Grosse, Exactly soluble system of relativistic twobody interaction, Phy. Rev. D, 16 (1977), 1900-1911.

[3] A. ARAi, On a model of a harmonic oscillator coupled to a quantized, massless, scalar field. I, II, J. Math. Phys., 22 (1981), 2539-2548 2549-2548.

[4] J.D. Bjorken AND S.D. Drell, Relativistic quantum fields, McGraw-Hill, New York, 1965.

[5] R.H. Cameron, A family of integrals serving to connect the Wienner and Feynman integrals, J. Math. Phys., 39 (1960), 126-140.

[6] M.D. DonsKar AND J.L. Lions, Fréchet-Volterra variational equations, boundary value problems and function space integrals, Acta Math., 108 (1962), 147-228.

[7] C. Folas, Statistical study of Navier-Stokes equations I, Rend. Sem. Mat. Padova, 48 (1973), 219-349.

[8] C. Folas, Statistical study of Navier-Stokes equations Il, Rend. Sem. Mat. Padova, 49 (1973), 9-123.

[9] I. M. GeLFAnd, Some aspects of functional analysis and algebra, International Math. Congress in Amsterdam, 1952.

[10] I. M. Gelfand and G. E. Shilov, Generalized functions, Vol. 1, Academic Press, New York, 1964.

[11] E. Hopf, Statistical hydrodynamics and functional calculus, J. Rat. Mech. Anal., 1 (1952), 87-123.

[12] A. Inoue, An explicit solution of a certain Schwinger-Dyson equation, Proc. Japan Acad. Ser A., 57 (1981), 477-480.

[13] C. ITzykson And J.B. Zuber, Quantum field theory, McGraw-Hill, New York, 1980.

[14] P. LEVy, Probleme concrets d'analyse fonctionnelle, Gauthier-Villars, Paris, 1951.

[15] R.E. Norton and W.K.R. Watson, Commutation rules and spurious eigenstates in soluble field theories, Phys. Rev., 116 (1959), 1597-1603.

[16] K. RzAZEWSKI AND W. ZAKOWICZ, Initial value problem and causality of radiating oscillator, J. Phys. A : Math. Gener., 9 (1976), 1159-1170.

[17] F. Schwahbl and W. ThirRing, Quantum theory of laser radiation, Erg. Exact Nat., 36 (1964), 219-242.

[18] L. Schwartz, Theorie des distributions, I, II, Hermann, Par1s, 1950-51.

[19] V. Volterra, Lecons sur les equations de lignes, Gauthier-Villars, Paris, 1913.

[20] F. Zachariasen, Relativistic model field theory with finite self masses, Phy. Rev., 121 (1961), 1851-1862.

DepartmeNT OF MATHEMATICS,

Tokyo Institute of Technologr, Oh-okayama, Meglio-ki; Tokyo, 152 ,

JAPAN 